\title{
Clases de grupos topológicos que se pueden encajar como subgrupos de productos de grupos primero numerable y segundo numerable
}

\author{
Adolfo Javier Pimienta Acosta
}

Tesis de Maestría

Asesor:

Constancio Hernández García

Universidad Autónoma Metropolitana

División de Ciencias Básicas e Ingeniería

Departamento de Matemáticas

Noviembre de 2011 
Matricula: 209382532

CLASES DE GPUPOS TOPOLOGICOS

QUE SE PUEDEN ENCAJAR COMO SUBGRUPOS DE PRODUCTOS DE

GRUPOS PRIMERO NUMERABLES O

SEGUNDO NUMERABLES

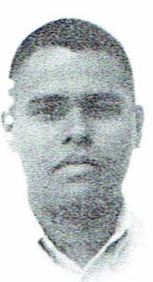

$+d y$ fo Pimidrita ADOLFO JAVIER PIMIENTA ACOSTA ALUMNO
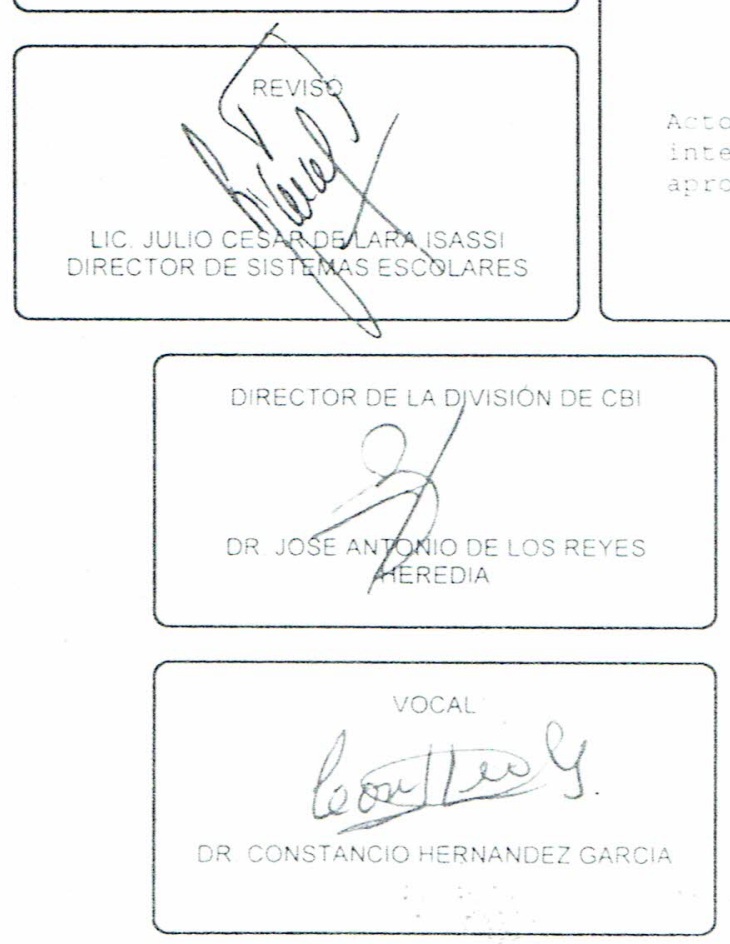

En México, D.F., se presentaron a las 25:00 hovas del dia 16 del mes de noviembre del año 2021 en la Unidad Iztapalapa de la Universidad Autónoma Kecropolitana. los suscritos miembros del jurado:

DR. ADALBERTO GARCIA MAYNEZ Y CERVANTES

DR. CONSTANCIO HERNANDEZ GARCIA

DR. RICHARD GORDON WILSON ROBERTS

Bajola presidencia del primero $y$ con cactcos de secrecario el utcimo, se reunieron para proceder i. Examen de Grado cuya denominación aparece al margen para la obtención del grado de:

WAESTRO EN CIENCIAS MATEMÁTICAS

DE: ADOIFO JAVIER PIMIENTA ACOSTA

$y$ de acuerdo con el articulo 78 zraccion It del Reglamento de Estudios Superiores de ia Universidad Autónoma Metropolitana, los miembros del quado resolvieron:

$$
\text { APROBAR }
$$

Acto continuo, el presidente del jurado comunicó al interesado el resultado de la evaluación y en caso aprobatorio, le fue comada la protesta. 


\title{
Dedicatoria
}

\author{
A Ana Victoria Guillen Bassa \\ aunque físicamente no estés conmigo, siempre \\ estarás en mi corazón y en mi mente
}




\section{Agradecimientos}

Antes que nada quiero agradecerle a Dios, Padre Todopoderoso, por darme la sabiduría para alcanzar una meta más en mi vida.

Son muchas las personas especiales a las que me gustaría agradecer su amistad, apoyo, ánimo y compañía en las diferentes etapas de mi vida. Algunas están conmigo y otras en mis recuerdos y en mi corazón. Sin importar en dónde estén o si alguna vez llegan a leer estas palabras quiero darles gracias por formar parte de mí, por todo lo que me han brindado y por todas su bendiciones.

Mami, no me equívoco si digo que eres la mejor mamá del mundo, gracias por todo tu esfuerzo, tu apoyo y por la confianza que depositaste en mí. Gracias porque siempre, aunque lejos, has estado a mi lado. Te quiero mucho.

Papi, este es un logro que quiero compartir contigo, gracias por creer en mí.

A mis hermanos: Danys y Jorge por estar siempre conmigo.

A ti margui, mi futura esposa, por apoyarme en esto y por las porras que me echas siempre. Te amo preciosa.

A la universidad Autónoma Metropolitana-Iztapalapa, en particular, al departamento de Matemáticas y a sus profesores, en especial a los doctores: Luis Miguel Villegas Silva, Mario Pineda Ruelas y Joaquín Delgado Fernández, por haberme hecho parte de esta casa.

A las secretarias del departamento de Matemáticas, que me recibieron con una sonrisa cada vez que fui a visitarlas

Al Dr. Constancio Hernández García, quien sin conocerme, aceptó asesorar este trabajo y guiarme en el largo camino de la topología general. Gracias por su apoyo en todos los sentidos, sus enseñanzas, su orientación y sobre 
todo su paciencia.

A los Doctores Adalberto García-Maynez y Richard Gordon Wilson por haberse tomado la molestia de leer este trabajo, además de los comentarios y observaciones que hicieron para la mejora de la misma. Les reitero mi profunda admiración.

Al Concejo Nacional de Ciencia y Tecnología (CONACYT), por darme la oportunidad de poder mantenerme en México con su beca. A la Maestra María Iseo, por la voluntad que siempre tuvo para hacer las cosas y siempre darme una solución a todo, a México por la oportunidad que me dio, por eso siempre serás México lindo y querido.

A mis compañeros del seminario de grupos topológicos, en especial, a Manuel Fernández Villanueva Medina y al Dr. Leonardo Rodríguez por todos sus comentarios y su ayuda.

A todos mis compañeros de la sala de posgrado, en especial, a Henry Chimal y a José Luís Cosme por la ayuda brindada.

A mi gran amigo en México Juan José Bernal por toda su ayuda.

A mi prima Esmeralda Fuentes, a las señoras Rocío y Rosaura Rivera, porque siempre estuvieron cuando lo necesité.

A todos mis amigos de Colombia, en especial, a Johnny Cuadro Molina, Johnny Rinaldi Villalobos y Leonardo Cortina Jaramillo. A los profesores Oswaldo Dede Mejía, Joaquín Luna Torres y Gilberto López Caraballo (Q.E.P.D), quienes me siguieron ayudando, incluso cuando ya no era su alumno. A mi gran amigo Ricardo Navarro Torres, por todo lo que ha hecho por mí, gracias pello.

En general, agradezco a todos los que no mencioné aquí pero que de alguna forma aportaron para que este sueño, mi sueño se hiciera realidad.

A todos los que acabo de mencionar les digo: Muchas Gracias. 


\section{Índice general}

\begin{tabular}{|l|l|}
\hline Agradecimientos & I
\end{tabular}

Introducción IV

0.1. Objetivos ............................ 1

0.1 .1$. General . . . . . . . . . . . . . . . . 1

$0.1 .2 . \quad$ Específicos . . . . . . . . . . . . . . . 1

1. Preliminares 2

1.1. Funciones Cardinales . . . . . . . . . . . . . . . . . 3

1.2. Propiedades de los grupos topológicos . . . . . . . . . . . . 4

1.3. Prenormas en grupos topológicos, metrización . . . . . . . . 10

2. Grupos Topológicos $\omega$-estrechos 13

2.0.1. Definición y Propiedades básicas. . . . . . . . . . . . 13

$\begin{array}{ll}\text { 3. Grupos Topológicos } \omega \text {-balanceados } & 17\end{array}$

3.0.2. Definición y Propiedades básicas. . . . . . . . . . . . 17

4. Conclusiones y futuras Direcciones 28

A. Acciones de Grupos Topológicos sobre Espacios Topológicos 30

A.1. Espacios Dugundji y funciones de suavidad-cero . . . . . . . . 30

A.2. Acciones continuas de grupos topológicos sobre espacios. . . . 33

A.3. Teoremas de Uspenskij's sobre acciones transitivas continuas de grupos $\omega$-estrechos sobre compactos $\ldots \ldots \ldots . . \ldots 35$ 


\section{Introducción}

Esta tesis se sitúa en el área de los grupos topológicos. La teoría de los grupos topológicos nace como un intento de enlazar dos ramas de la matemáticas: la teoría (algebraica) de los grupos y la topología general. Durante los últimos años, la teoría de los grupos topológicos ha motivado numerosas investigaciones. Por una parte se han incorporado varios resultados algebraicos, como los teoremas de estructuras, lo que ha permitido por ejemplo, la descripción de la estructura de los grupos abelianos localmente compactos. Por otro lado, la revolución que han significado en la topología general los recientes resultados en la teoría de conjuntos también se ha reflejado en la teoría de los grupos topológicos, pues las nuevas técnicas y métodos de trabajo han propiciado que se revelen propiedades muy sutiles de los grupos topológicos, que se resuelvan problemas que permanecieron sin solución por largo tiempo y que se abran nuevas lineas de investigación. Los espacios Tychonoff son espacios que se pueden encajar en un producto topológico de la forma $I^{\mathfrak{m}}$, donde $\mathfrak{m}$ es un cardinal suficientemente grande e $I=[0,1]$. Queremos tener una opción de clasificación semejante en grupos topológicos. En este caso, como tenemos estructura adicional (la algebraica) no podemos utilizar ni $I$ ni $\mathbb{R}$. Recurrimos entonces a grupos segundo numerables o grupos primero numerables. Por el teorema de Birkoff-Kakutani, un grupo topológico es primero numerable si, y sólo si, es metrizable. Diremos que un grupo topológico $G$ es $\omega$-balanceado si el número de invariancia (denotado por $i n v(G) \leq \omega$ ) es numerable, esto es si para toda vecindad $U$ de la identidad de $G$, existe una familia numerable $\gamma$ de vencindades de la identidad en $G$ tal que para todo $x \in G$, existe $V \in \gamma$ que satisface $x V x^{-1} \subset U$. Si tal familia $\gamma$ existe se llamará subordinada a $U$. Claramente todo subgrupo de un grupo $\omega$-balanceado en $\omega$-balanceado. 
Un grupo topológico es $\omega$-balanceado si, y sólo si, se puede encajar como subgrupo de un producto de grupos primero numerables. En este trabajo nos damos a la tarea de estudiar las clases de los grupos topológicos $\omega$-estrechos y $\omega$-balanceados. La clase de los grupos $\omega$-balanceados es muy amplia. De hecho, no es fácil hallar un ejemplos de un grupo que no lo sea. Daremos un ejemplo de un grupo topológico que no es $\omega$-balanceado. Diremos que un grupo topológico $G$ es $\omega$-estrecho si para toda vecindad $U$ de la identidad de $G$ existe un conjunto numerable $V \subseteq G$ tal que $G=V \cdot U$. El teorema de Guran establece que un grupo topológico es $\omega$-estrecho si, y sólo si, se puede encajar como subgrupo de un producto de grupos segundo numerables. La clase de los grupos $\omega$-estrechos es muy interesante porque es muy estable bajo las operaciones más frecuentes sobre los grupos topológicos. Es decir, es cerrada bajo subgrupos y bajo productos. 


\section{Resumen histórico}

La teoría de los grupos topológicos es uno de los ejemplos más interesantes de interacción exitosa de dos áreas distintas de las matemáticas: la teoría de los grupos y la topología general. A través de esta última se incorpora también otra rama fundamental, como la teoría de conjuntos. La convergencia de éstas fue el resultado de la influencia de la teoría de los grupos de Lie y de varias clases de grupos de transformaciones. Es, por tanto, difícil, delimitar la frontera entre la topología general y otras diciplinas próximas. Por ejemplo, algunas cuestiones planteadas en campos limítrofes pueden ser abordadas y resueltas con técnicas y conceptos que surgen de la topología general. Este fenomeno ha sido (y es) relevante y ha estado motivado con el hecho de que muchos investigadores en topología general se han formado en áreas colindantes como el análisis funcional o la geometría, e incluso el álgebra.

Uno de los ejemplos de esta dificultad a la hora de establecer la frontera entre la topología general y otras materias son los grupos topológicos abstractos, los cuales fueron definidos por Schreier en 1926, aunque la idea estaba implícita en trabajos muy anteriores sobre grupos continuos de transformaciones. La materia tiene sus orígines en e programa de Klein (1872) de estudiar geometrías atraves de los grupos de tranformaciones asociadas a ellos y la teoría de Lie de grupos continuos saliendo de la solución de ecuaciones diferenciales. Los grupos clásicos de la geometría (grupos lineales generales, grupos unitarios, grupos simpléticos...) son de hecho, grupos de Lie, es decir, son variedades analíticas y sus operaciones de grupos son funciones analíticas. Por otra parte, Killing y Cartan probaron que todos los grupos de Lie simples 
son grupos clásicos, excepto un número finito de grupos excepcionales. En relación con los grupos topológicos, en 1900, Hilbert propuso el problema, el que hacía el número 5 de su famosa lista, de si todo grupo continuo de transformaciones de un espacio real o complejo de dimensión finita es un grupo de Lie. Sin embargo, este problema acabó formulandose de una forma más abstracta. Un grupo topológico es un espacio topológico con las operaciones de grupo continuas, y la pregunta es: ¿ qué condiciones topológicas sobre un grupo topológico aseguran que tenga una estructura analítica que hagan que sea grupo de Lie?. Como la integración era una herramienta fundamental en el estudio de los grupos de Lie, especialmente en las representaciones, el establecimiento de la existencia de las integrales apropiadas sobre clases generales de grupos topológicos se convertió en una cuestión importante. Esto lo consiguio Haar en 1933 para grupos localmente compactos con bases de abiertos numerables. Von Neumann (1934) dió otra prueba para grupos compactos arbitrarios de manera que la teoría de grupos de Lie compactos podía aplicarse a todos los grupos compactos y pudo resolver en este caso especial el problema planteado por Hilbert. El método de Haar de integración fue extendido a todos los grupos localmente compactos por Weil (1940). Sin embargo, existen serios obstaculos a la hora de extender la teoría de representación a grupos localmente compactos, y no fue hasta 1952 cuando el problema de Hilbert fue asentado por Gleason, Montgomery y Zippin. Su respuesta puede formularse de la siguiente forma: un grupo topológico es un grupo de Lie si, y sólo si, es localmente compacto y no tiene subgrupos arbitrarios pequeños, es decir, el elemento identidad tiene un entorno compacto que no contiene subgrupos no triviales.

Y, aunque la teoría de los grupos topológicos se desarollo principalmente para estudiar los grupos de Lie su impulso provino de problemas en análisis, pronto se probó que era útil en conceptos puramente algebraicos. Ciertas construcciones algebraicas hacen que los grupos tengan estructuras topológicas naturales que son de alguna forma patólogicas desde el punto de vista del analista. Como ejemplo tenemos los anillos de series de potencias, los grupos de Galois de extensiones infinitas de cuerpos y los grupos p-ádicos. Dicha patología se sitúa en la existencia de subgrupos arbitrariamente pequeños, pero en los casos más importantes los grupos son, de hecho, localmente compactos y de ahí que la integración pueda llevarse acabo sobre ellos.

La clase de los grupos topológicos $\omega$-estrechos fue introducida por I. I. Guran en 9, no siempre fuerón llamados $\omega$-estrechos anteriormente eran conocidos 
como $\aleph_{0}$-acotados. Dado que este concepto ya era utilizado en espacios topológicos, se decidio cambiar la terminología y llamar a los grupos en cuestión $\omega$-estrechos.

Estudiando está clase de grupos topológicos se pudo observar que gozaban de propiedades muy importantes, eran cerrados bajo productos, bajo subgrupos y bajo imagenes de homomorfismos continuos. Esto mostraba que la clase de los grupos topológicos $\omega$-estrechos era análoga o paralela a la clase de espacios Tychonoff. De hecho, la clase más pequeña de espacios topológicos que contiene todos los espacios metrizables separables, cerrada bajo subespaciosy productos arbitrarios, es la clase de los espacios Tychonoff.

La clase de los grupos topológicos $\omega$-estrechos, caracteriza a los grupos topológicos que se pueden encajar como un subgrupo de un producto de grupos segundo numerable.

Por otro lado, la clase de los grupos topológicos $\omega$-balanceados fue introducida por G. I. Kats en 11, está clase de grupos topológicos caracterizaba a la clase de grupos topológicos que se pueden encajar como un subgrupo de un producto de grupos metrizables. 


\subsection{Objetivos}

\subsubsection{General}

Presentar un estudio detallado de la clase de los grupos $\omega$-estrechos y de la clase de los grupos $\omega$-balanceados dando caracterizaciones internas que nos aclaren los aspectos más importantes de la estructura de estos dos tipos de grupos topológicos. Además, describiremos las propiedades más importantes de estas familias de grupos enriqueciendo con ejemplos el alcance y pertinencia de los teoremas.

\subsubsection{Específicos}

- Comenzaremos con los grupos $\omega$-estrechos, dando su definición y probaremos que se pueden caracterizar como grupos que se pueden encajar en producto de grupos segundo numerables.

- Estudiaremos los grupos topológicos $\omega$-balanceados y sus propiedades más importantes.

- Daremos un ejemplo, de un grupo topológico que no es $\omega$-balanceado. 
canowat 1

\section{Preliminares}

En este apartado se establecen algunas definiciones y notaciones básicas, necesarias para la comprensión de este proyecto. Todas la demostraciones se omiten. En cambio, el lector encotrará la referencia donde se demuestran los resultados mencionados. Todos los resultados se encuentran en 7 Recordemos que si $(X, \tau)$ es un espacio topológico que posee una base numerable $\mathcal{B}$, se dice que $X$ es segundo numerable. Si $(X, \tau)$ es un espacio topológico tal que todo punto $x \in X$ posee una base local en $x$ numerable, decimos que $X$ es un espacio primero numerable. Un espacio $X$ Hausdorff es $T_{3 \frac{1}{2}}$ o Tychonoff si para todo $x \in X$ y todo cerrado $F \subseteq X$ tal que $x \notin F$ existe una función continua $f: X \rightarrow[0,1]$ con la propiedad que $f(x)=0$ y $f(y)=1$ para todo $y \in F$. Todo espacio segundo numerable y regular es normal. Lo mismo ocurre con todo espacio regular y numerable.

Definición 1.1. Sean $X$ un espacio y $\left\{f_{i}: i \in I\right\}$ un conjunto de funciones tales que $f_{i}: X \rightarrow Y_{i}$. Definimos el producto diagonal $f: X \rightarrow \prod_{i \in I} Y_{i}=$ $\Delta_{i \in I} f_{i}$ mediante la fórmula $f(x)=\left(f_{i}(x)\right)_{i \in I}$.

Definición 1.2. Sea $X$ un espacio topológico, $\left\{Y_{\alpha}\right\}_{\alpha \in S}$ una familia de espacios topológicos, sea $\mathcal{F}=\left\{f_{\alpha}\right\}_{\alpha \in S}$ una familia de funciones continuas, donde $f_{\alpha}: X \rightarrow Y_{\alpha}$. Decimos que $\mathcal{F}$ separa puntos si dados $x, y \in X$, con $x \neq y$ existe $f_{\alpha} \in \mathcal{F}$ tal que $f_{\alpha}(x) \neq f_{\alpha}(y)$. Además si para todo $x \in X$, y todo conjunto cerrado $F \subset X$ tal que $x \notin F$, existe una $f_{\alpha} \in \mathcal{F}$ tal que $f_{\alpha}(x) \notin \overline{f_{\alpha}(F)}$, entonces decimos que la familia $\mathcal{F}$ separa puntos de conjuntos cerrados.

Lema 1.3. Si la función continua $f: X \rightarrow Y$ es uno a uno y la familia de 
un elemento $\{f\}$ separa puntos y conjuntos cerrados, entonces $f$ es un encaje homeomorfico.

El siguiente teorema describe una forma de obtener encajes de espacios en productos cartesianos.

Teorema 1.4 (Teorema de la Diagonal). Si $\mathcal{F}=\left\{f_{i}: X \rightarrow Y_{i}: i \in I\right\}$ es una familia de funciones continuas que separa puntos de $X$, entonces la función diagonal $f=\Delta_{i \in I} f_{i}$ es inyectiva. Más aún, si la familia $\mathcal{F}$ separa puntos de cerrados de $X$, entonces $f$ es un encaje homeomorfico.

\subsection{Funciones Cardinales}

En esta sección definimos la funciones cardinales mas conocidas.

Definición 1.5. Sea $X$ un espacio topológico, definimos:

- El peso de $X$, como $w(X)=\operatorname{mín}\{|\mathcal{B}|: \mathcal{B}$ es una base de $\mathrm{X}\}+\aleph_{0}$.

- La densidad de $X, \operatorname{como} d(X)=\operatorname{mín}\{|D|: D$ es un subconjunto denso de $\mathrm{X}\}+$ $\aleph_{0}$.

- Una familia de abiertos no vacíos mutuamente ajenos es una familia celular. La celularidad de $X$, esta definida por, $c(X)=\min \{|\mathcal{V}|: \mathcal{V}$ es una familia celular en $\mathrm{X}\}+\aleph_{0}$

- La noción de espacio de Lindelöf da lugar a una nueva función cardinal, el número de Lindelöf de un espacio $X$, denotado por $l(X)$ es el número cardinal $\kappa$ más pequeño tal que toda cubierta abierta de $X$ tiene una subcubierta de cardinalidad no mayor que $\kappa$.

- El carácter de un punto $x$ en un espacio topológico $X$ está definido por $\chi(x, X)=\operatorname{mín}\{|\mathcal{B}|: \mathcal{B}$ es una base local en $\mathrm{x}\}$; definimos el carácter como sigue: $\chi(X)=\sup \{\chi(x, X): x \in X\}+\aleph_{0}$.

- La estrechez $t(X)$ de $X$ es el cardinal infinito $\tau$ más pequeño tal que si $x \in \bar{A}$, donde $x \in X$ y $A \subset X$, entonces existe un $B \subset A$ para el cual $x \in \bar{B}$ y $|B| \leq \tau$. 


\subsection{Propiedades de los grupos topológicos}

El objetivo de esta sección es presentar un breve estudio de las propiedades elementales más importantes de los grupos topológicos. No pretendemos dar una introducción a los grupos topológicos, el objetivo es dar una referencia lo más completa posible a quien lea esta tesis sobre los resultados utilizados en la parte principal del trabajo, la cual se ubica en los capítulos 4 y 5. Las demostraciones de todos los resultados mencionados en este apartado puede ser consultada en 14 y 4 .

Definición 1.6. Un conjunto $G$ con una operación binaria · y una familia $\tau$ de subconjuntos de $G$ se llama grupo topológico si

1) $(G, \cdot)$ es un grupo;

2) $(G, \tau)$ es un espacio topológico;

3) Las funciones $\mu:(G, \tau) \times(G, \tau) \rightarrow(G, \tau)$ y $\iota:(G, \tau) \rightarrow(G, \tau)$ dadas por $\mu(x, y)=x \cdot y$ y $\iota(x)=x^{-1}$ son continuas, donde $x^{-1}$ es el inverso de $x$.

En ocasiones prescindiremos del uso del símbolo de operación binaria · es decir, en vez de $x \cdot y$ escribiremos simplemente $x y$.

Sea $G$ un grupo topológico, si denotamos con $\mathcal{N}(x)$ a la familia de vecindades de un punto $x \in G$, podemos describir la condición (3) de la definición 1.6 como sigue: si $x$ y $y$ son elementos de $G$, para cada $U \in \mathcal{N}(x y)$ existen vecindades $V \in \mathcal{N}(x)$ y $W \in \mathcal{N}(y)$ tales que $V \cdot W \subset U$, donde $V \cdot W=\{v w$ : $v \in V, w \in W\}$; y para cada $U \in \mathcal{N}\left(x^{-1}\right)$ existe $V \in \mathcal{N}(x)$ tal que $V^{-1} \subset U$, donde $V^{-1}=\left\{v^{-1}: v \in V\right\}$. El símbolo $e$ denotará siempre a la identidad de un grupo $G$.

Sea $G$ un grupo topológico. Para cada $g \in G$ fijo, las funciones $\varphi_{g}(x)=$ $x g$ y $\sigma_{g}(x)=g x, x \in G$ de $G$ en sí mismo, son homeomorfismos. La inversión $f: G \rightarrow G$, definida por $f(y)=y^{-1}$, también es un homeomorfismo. Las funciones $\varphi_{g}$ y $\sigma_{g}$ se llaman traslaciones por $g$ derecha e izquierda, respectivamente.

Observe que una consecuencia de que $\varphi_{g}$ y $\sigma_{g}$ sean homeomorfismos es que todo grupo topológico es un espacio homogéneo. Esto permitirá estudiar las propiedades locales de un grupo topológico $G$ en un solo punto, que por simplificar siempre tomaremos como la identidad $e$ de $G$. 
Describir la topología en un grupo topológico es generalmente una tarea más fácil que hacerlo es un espacio topológico arbitrario. Basta describir una base local para la identidad $e$ en el grupo $G$.

Lema 1.7. Sea $G$ un grupo topológico, y sea $\mathcal{N}(e)$ una base local para la identidad e del grupo $G$. Entonces las familias $\{x U\}$ y $\{U x\}$, donde $x$ toma valores en los elementos de $G$ y $U$ varía sobre todos los elementos de $\mathcal{N}(e)$, son bases para la topología del grupo $G$.

El lema siguiente nos proporciona una base local para la identidad formada por vecindades tales que $V^{-1}=V$. Estas vecindades reciben el nombre de simétricas.

Lema 1.8. Si $G$ es un grupo topológico y $U \in \mathcal{N}(e)$, entonces existe $V \in \mathcal{N}(e)$ una vecindad simétrica tal que $V \subseteq U$. Por lo tanto, las vecindades simétricas de la identidad e constituyen una base local para e en el grupo $G$.

La identidad de un grupo topológico tiene aún otra propiedad muy importante: admite una base local formada por subconjuntos cerrados.

Lema 1.9. Sea $G$ un grupo topológico.

(1) Si $U \in \mathcal{N}(e)$, entonces para cada $n \in \mathbb{N}^{+}$existe $V \in \mathcal{N}(e)$ con $V^{n} \subseteq U$ $\left(V^{n}=V \cdots V, n\right.$ factores $)$

(2) Si $U \in \mathcal{N}(e)$, entonces existe $V \in \mathcal{N}(e)$ con $\bar{V} \subseteq U$. En particular las vecindades cerradas de e en $G$ constituyen una base local para la identidad e cuyos elementos son subconjuntos cerrados.

El hecho de que las traslaciones en los grupos topológicos son homeomorfismos se utiliza para probar que el producto de un abierto por cualquier conjunto es abierto. En el caso de los subconjuntos cerrados tenemos una situación similar sólo para conjuntos compactos.

Lema 1.10. Sean $G$ un grupo topológico, $a \in G$ y $A, B, O, M$ subconjuntos de G. Entonces:

(1) Si $O$ es abierto, los conjuntos aO, Oa, $O^{-1}, M O, O M$ son abiertos.

(2) Si A es cerrado a $A, A a, A^{-1}$ son conjuntos cerrados.

(3) Si $A$ y $B$ son compactos, también lo son $A B$ y $A^{-1}$. 
Lema 1.11. Sea un grupo topológico $G$. Si $G$ es $T_{0}$, entonces es $T_{3 \frac{1}{2}}$.

El siguiente teorema es de suma importancia. En él se resumen varias de las propiedades obtenidas anteriormente para la familia $\mathcal{N}(e)$; de hecho, esta familia se caracteriza completamente. Ésta es una de las propiedades que distinguen a los grupos topológicos de los espacios topológicos arbitrarios. Además, dicho teorema nos proporciona un métod para definir topologías de grupos topológicos.

Teorema 1.12. Sea $G$ un grupo topológico Hausdorff. Existe una base local $\mathcal{V}$ para e en $G$ que cumple las siguientes condiciones.

(1) $\bigcap \mathcal{V}=\{e\}$

(2) Si $U, V$ son dos elementos arbitrarios de $\mathcal{V}$, entonces existe $W \in \mathcal{V}$ tal que $W \subseteq U \cap V$;

(3) para cada $U \in \mathcal{V}$ existe $V \in \mathcal{V}$ tal que $V V^{-1} \subseteq U$;

(4) para cada $U \in \mathcal{V}$ y para cada $x \in U$ existe $V \in \mathcal{V}$ con $x V \subseteq U$;

(5) para cada $U \in \mathcal{V}$ y $a \in G$ existe $W \in \mathcal{V}$ con $a W a^{-1} \subseteq U$.

Recíprocamente, si tenemos un grupo y una familia $\mathcal{V}$ no vacía de subconjuntos de $G$ que contienen a e, tal que se saisfacen las condiciones $(1)-(5)$ para $\mathcal{V}$, entonces cada una de las familias $\{x U: U \in \mathcal{V}, x \in G\}$ y $\{U x: U \in \mathcal{V}, x \in G\}$ es base para una topología de grupo $\tau$ para $G$. Además $\mathcal{V}$ es una base local para e en $(G, \tau)$.

Ejemplo 1.13. 1. Sea $G$ un grupo arbitrario, y sea $\mathcal{T}$ la familia de todos los subconjuntos de $G$ [la topología discreta]. Con esta topología, $G$ es un grupo topológico

2. Sea $G$ un grupo arbitrario, y sea $\mathcal{T}$ consistente de $\varnothing$ y $G$ solamente. Entonces $G$ es un grupo topológico.

3. El grupo aditivo $\mathbb{R}$ de todos los números reales con su topología usual.

4. El grupo lineal general de orden $n$ sobre $\mathbb{R}$. Considere el grupo $G L(n, \mathbb{R})$ de las matrices no singulares (invertibles) de orden $n$, con la topología inducida de $\mathbb{R}^{n^{2}}$. 
El lector con experiencia en análisis funcional recordará que basta probar la continuidad de un operador lineal en la identidad de un espacio vectorial topológico para saber que es continuo en todo el espacio. Esta propiedad es aún válida para grupos topológicos.

Lema 1.14. Sea $\varphi: G \rightarrow H$ un homomorfismo entre grupos topológicos. El homomorfismo $\varphi$ es continuo (respectivamente abierto) si lo es en la identidad $e_{G}$, es decir, si $\varphi$ satisface la condición (1) (respectivamente 2) siguiente:

1) Para toda $W$ vecindad de $e_{H}$ en $H$, existe $U$ vecindad de $e_{G}$ en $G$ tal que $\varphi(U) \subseteq W$;

2) para toda vecindad $U$ de $e_{G}$ en $G$, existe $W$ vecindad de $e_{H}$ tal que $W \subseteq \varphi(U)$.

De manera similar al caso de subespacios topológicos, la operación de tomar subgrupos topológicos es una fuente muy importante para construir "nuevos" grupos topológicos a partir de los ya conocidos.

Definición 1.15. Sea $G$ un grupo topológico, un subconjunto $H$ de $G$ se llama subgrupo topológico de $G$ si

(1) $H$ es un subgrupo del grupo $G$;

(2) $H$ es un subespacio con la topológia inducida de $G$.

El siguiente resultado justifica la definición de subgrupo topológico en el sentido de que este ultimo es por sí mismo un grupo topológico.

Proposición 1.16. Sean $G$ un grupo topológico y $H$ un subgrupo topológico de $G$. Entonces $H$ es un grupo topológico con la topología que hereda de $G$.

Recordemos que un subgrupo $H$ de un grupo $G$ se llama subgrupo normal de $G$ si $a^{-1} H a \subseteq H$ para todo $a \in G$.

Proposición 1.17. Sean $G$ un grupo topológico y $H, N$ subgrupos de $G$. Entonces:

(1) $\bar{H}$ es un subgrupo de $G$;

(2) Si $N$ es un subgrupo normal de $G$, entonces $\bar{N}$ es un subgrupo normal de $G$; 
(3) H es abierto si y sólo si su interior no es vacío;

(4) Si $H$ es abierto, entonces $\bar{H}=H$.

Pasemos ahora al estudio de grupos cocientes. Definiremos una topología de grupo en el conjunto de clases laterales.

Definición 1.18. Sea $G$ un grupo topológico, y $H$ un subgrupo de $G$. Sea $\pi: G \rightarrow G / H$ el homomorfismo canónico de $G$ sobre $G / H$. Definimos una topología $\mathcal{O}(G / H)$ por la siguiente regla: un subconjunto $V \subseteq G / H$ esta en $\mathcal{O}(G / H)$ si y sólo si $\pi^{-1}(V)=U$, donde $U$ es abierto en $G$.

Dado que $G / H=\{H x: x \in G\}$, se sigue que todo conjunto abierto en $G / H$ tiene la forma $V=\{H u: u \in U\}$ donde $U$ es abierto en $G$. Por lo tanto $\mathcal{O}(G / H)=\{V \in G / H: U \in G\}$. El espacio topológico $G / H$ así construido recibe el nombre de espacio cociente de $G$ entre $H$.

Proposición 1.19. Sean $G$ un grupo topológico, $H$ un subgrupo cerrado de $G$ y $\pi: G \rightarrow G / H$ la función canónica. Entonces $\pi$ es continua y abierta.

El resultado siguiente nos muestra que las propiedades topológicas locales del espacio cociente $G / H$ se pueden estudiar en un solo punto.

Proposición 1.20. Sean $G$ un grupo topológico y $H$ un subgrupo cerrado de $G$; entonces $G / H$ es un espacio homogéneo.

El siguiente resultado es un lema auxiliar que nos permitirá establecer dos propiedades topológicas del espacio cociente $G / H$.

Lema 1.21. Sean $G$ un grupo topológico, $H$ un subgrupo cerrado de $G$ y $U, V$, vecindades abiertas de en $G$ tales que $V V^{-1} \subseteq U$. Entonces $\pi: G \rightarrow G / H$ es la función canónica, se cumple que $\overline{\pi(V)} \subseteq \pi(U)$.

Teorema 1.22. Sean $G$ un grupo topológico y $H$ un subgrupo cerrado de $G$. Entonces

(1) $G / H$ es un espacio regular y por lo tanto Hausdorff.

(2) $G / H$ es un espacio discreto si y sólo si $H$ es abierto en $G$.

En el caso particular en el que subgrupo $H$ de $G$ es normal, de modo que $G / H$ es un grupo, se tiene el siguiente resultado. 
Teorema 1.23. Sean $G$ un grupo topológico y $H$ un subgrupo normal y cerrado de $G$, entonces

1) $G / H$ con la topología cociente es un grupo topológico;

2) la función canónica $\pi: G \rightarrow G / H$ es un homomorfismo abierto y continuo;

3) el grupo $G / H$ es un espacio $T_{1}$ y por tanto regular.

4) el grupo $G / H$ es discreto si y sólo si $H$ es abierto.

Sea $\left\{G_{i}\right\}_{i \in I}$ una familia de grupos topológicos. Damos estructura de grupo al producto cartesiano $G=\prod_{i \in I}$ definiendo $\left(x_{i}\right)_{i \in I}\left(y_{i}\right)_{i \in I}=\left(x_{i} y_{i}\right)_{i \in I}$. Si $e_{i}$ es elemento identidad de $G_{i}$, entonces $e=\left(e_{i}\right)_{i \in I}$ es elemento identidad de $G$, y tenemos $\left(x_{i}\right)_{i \in I}^{-1}=\left(x_{i}^{-1}\right)_{i \in I}$. La topología producto de Tychonoff es compatible con esta estructura de grupo porque la función $h: G \times G \rightarrow$ $G$ dada por $h\left(\left(x_{i}\right)_{i \in I},\left(y_{i}\right)_{i \in I}\right)=\left(x_{i} y_{i}^{-1}\right)_{i \in I}$ es la composición de las funciones $\left(x_{i} y_{i}^{-1}\right)_{-1} \rightarrow\left(x_{i} y_{i}^{-1}\right)_{i \in I}$ de $\prod_{i \in I}\left(G_{i} \times G_{i}\right)$ en $G$ y la proyección $\left(\left(x_{i}\right)_{i \in I},\left(y_{i}\right)_{i \in I}\right) \rightarrow\left(\left(x_{i}, y_{i}\right)_{i \in I}\right)$ de $G \times G$ en $\prod_{i \in I}\left(G_{i} \times G_{i}\right)$, y estas dos funciones son continuas.

Definición 1.24. El producto cartesiano de una familia de grupos topológi$\cos \left\{G_{i}: i \in I\right\}$ se obtiene al dar el producto

$$
G=\prod_{i \in I} G_{i}
$$

la topología producto de Tychonoff.

La proyección natural $\pi_{j}: G \rightarrow G_{j}$ con $j \in J$ definida por $\pi(x)=x_{j}$ para todo $x=\left(x_{i}\right)_{i \in I} \in G$ es un homomorfismo continuo. Este último hecho se deduce directamente de la topología producto. Más aún, la función $\phi_{j}: G_{j} \rightarrow G$ definida por $\phi_{j}(x)=\left(y_{i}\right)_{i \in I}$, donde $y_{i}=e_{i}$ para $i \neq j$ y $y_{j}=x$, es un isomorfismo topológico entre $G_{j}$ y $N_{j}=\phi_{j}\left(G_{j}\right)$. Es decir, $\phi_{j}$ es un encaje de $G_{j}$ en $G$.

Definición 1.25. Decimos que un grupo $G$ es $\mathbb{R}$-factorizable si para toda función continua $f: G \rightarrow \mathbb{R}$ existen un homomorfismo continuo $\pi: G \rightarrow K$ de $G$ sobre un grupo topológico segundo numerable $K$ y una función continua $h: K \rightarrow \mathbb{R}$ tales que $f=h \circ \pi$. En esta definición, podemos sustituir los 
números reales por cualquier espacio $X$ regular y segundo numerable, dándonos, así, la posibilidad de factorizar funciones continuas $f: G \rightarrow X$ mediante homomorfismos continuos sobre grupos topológicos segundo numerables. Representemos esta definición con el siguiente diagrama conmutativo.

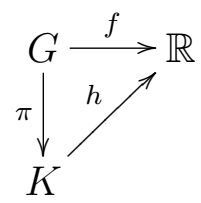

\subsection{Prenormas en grupos topológicos, metri- zación}

En esta sección estudiaremos el concepto de seudonorma en grupos topológicos. El objetivo será caracterizar la familia de vecindades de la identidad en terminos de prenormas. Todas la demostraciones se omiten. Las demostraciones pueden ser consultadas en su totalidad en 14 y 4 .

Definición 1.26. Sea $G$ un grupo con elemento identidad $e$ y sea $N$ una función de valores reales nonegativos definida en $G$. Diremos que $N$ es una prenorma en $G$ si las condiciones siguientes se cumplen para todos $x, y \in G$ :

(PN1) $N(e)=0$

(PN2) $N\left(x y^{-1}\right) \leq N(x)+N(y)$;

(PN3) $N\left(x^{-1}\right)=N(x)$.

Proposición 1.27. Si $N$ es una prenorma sobre $G$, entonces $N(x) \geq 0$ para cada $x \in G$, esto es, $N$ es no negativa.

Proposición 1.28. Si $N$ es una prenorma sobre un grupo $G$, entonces $|N(x)-N(y)| \leq N\left(x^{-1} y\right)$, para cada $x, y \in G$.

Proposición 1.29. Para cualquier prenorma $N$ sobre un grupo $G$, el conjunto $Z_{N}=\{x \in G: N(x)=0\}$ es un subgrupo de $G$.

Proposición 1.30. La suma de dos prenormas en un grupo $G$ es una prenorma en $G$. 
Existe un método muy sencillo para construir prenormas en grupos topológicos. Y es descrito en el siguiente ejemplo.

Ejemplo 1.31. Sea $f$ una función de valores reales en un grupo $G$. Entonces la función $N_{f}$ en $G$, definida por la formula:

$$
N_{f}(x)=\sup \{|f(y x)-f(y)|: y \in G\},
$$

para cada $x \in G$, es una prenorma en $G$.

Definición 1.32. Sean $G$ un grupo topológico y $N$ una prenorma en $G$. Decimos que $N$ es una prenorma invariante si $N(x)=N\left(y^{-1} x y\right)$ para cualquier $x$ y $y$ en $G$.

En general, una prenorma en un grupo topológico no tiene que ser continua. La siguiente proposición aunque simple es muy útil.

Proposición 1.33. Una prenorma $N$ en un grupo topológico $G$ es continua si y sólo si para todo $\epsilon>0$ existe una vecindad $U$ de la identidad e tal que $N(x)<\epsilon$, para todo $x \in U$.

Definición 1.34. Si $N$ es una prenorma sobre un grupo $G$, definimos la bola unitaria de $N$ como el conjunto $B_{N}=\{x \in G: N(x)<1\}$. Claramente, si $N$ es una prenorma continua, entonces la bola unitaria $B_{N}$ es un subconjunto abierto de $G$.

Teorema 1.35 (A. A. Markov). Para toda vecindad abierta $U$ de la identidad e de un grupo topológico $G$, existe una prenorma continua $N$ en $G$ tal que la bola unitaria $B_{N}$ esta contenida en $U$.

Lema 1.36. Sea $\left\{U_{n}: n \in \omega\right\}$ una sucesión de vecindades abiertas y simétricas de la identidad e en un grupo topológico $G$ tal que $U_{n+1}^{2} \subset U_{n}$, para cada $n \in \omega$. Entonces existe una prenorma $N$ sobre $G$ tal que la siguiente condición se cumple:

$$
(P N 4) \quad\left\{x \in G: N(x)<1 / 2^{n}\right\} \subset U_{n} \subset\left\{x \in G: N(x) \leq 2 / 2^{n}\right\} .
$$

Por lo tanto, esta prenorma $N$ es continua. Si, además, los conjuntos $U_{n}$ son invariantes, entonces en la prenorma $N$ sobre $G$, se pueden elegir para satisfacer $N\left(x y x^{-1}\right)=N(y)$ para todo $x, y \in G$. 
Definición 1.37. Una métrica en un conjunto $X$ es una función $d: X \times X \rightarrow$ $\mathbb{R}$ con las propiedades:

a) $d(x, y) \leq 0$ para toda $x, y \in X$.

b) $d(x, x)=0$ para toda $x \in X$.

c) Si $x, y \in X, x \neq y$, entonces $d(x, y) \neq 0$.

d) $d(x, z) \leq d(x, y)+d(y, z)$ para toda $x, y, z \in X$.

Definición 1.38. Sea $G$ un grupo topológico con una métrica $\rho$. La métrica $\rho$ es invariante por la izquierda si la relación

$$
\rho(z x, z y)=\rho(x, y)
$$

se cumple para cualquier $x, y$ y $z$ elementos de $G$. De una manera similar se define una métrica invariante por la derecha. Una métrica en el grupo $G$ que es simultaneamente invariante por la izquierda y por la derecha recibe el nombre de métrica invariante.

Teorema 1.39 (G. Birkhoff, S. Kakutani). Un grupo topológico G es metrizable si y sólo si es primero numerable.

Corolario 1.40. Todo grupo topológico primero numerable admite una métrica derecho-invariante $\rho$ y una métrica izquierdo-invariante $\lambda$, ambas generan la topología original del grupo $G$. 
${ }_{\text {Capítulo }}$

\section{Grupos Topológicos $\omega$-estrechos}

\subsubsection{Definición y Propiedades básicas}

Definición 2.1. Un grupo topológico $G$ es $\omega$-estrecho si para toda vecindad $U$ de la identidad de $G$ existe un conjunto numerable $A \subseteq G$ tal que $G=A \cdot U$.

Proposición 2.2. Las siguientes condiciones son equivalentes para un grupo topológico $G$ :

1) G es w-estrecho;

2) Para toda vecindad abierta $V$ de la identidade en $G$, existe un conjunto numerable $B \subset G$ tal que $G=V B$;

3) Para toda vecindad abierta $V$ de la identidad e en $G$, existe un conjunto numerable $C \subset G$ tal que $C V=G=V C$.

Demostración. Es claro que tanto (1) y (2) se siguen de (3). Además, (3) se sigue de la conjunción de (1) y (2). Ya que las igualdades $G=A \cdot V$ y $G=V \cdot B$ implican que $C \cdot V=G=V \cdot C$, donde $C=A \cup B$. Es claro que el conjunto $C$ es numerable si lo son $A$ y $B$.

Solo nos falta demostrar que (1) y (2) son equivalentes. Sea $G$ un grupo topológico $\omega$-estrecho. Dado una vecindad abierta $V$ de $e$, podemos encontrar una vecindad abierta $U$ de $e$ tal que $U^{-1} \subset V$. Elejimos un conjunto numerable $A \subset G$ tal que $A \cdot U=G$. Entonces el conjunto numerable $B=A^{-1}$ satisface $G=G^{-1}=(A \cdot U)^{-1}=U^{-1} A^{-1} \subset V \cdot B$, esto es que $G=V \cdot B$. Esto prueba que $(1) \Rightarrow(2)$. Invirtiendo el argumento anterior, obtenemos $(2) \Rightarrow(1)$. Por lo tanto, las tres condiciones son equivalentes. 
Proposición 2.3. Si un grupo topológico $H$ es imagen homomorfica continua de un grupo topológico $G \omega$-estrecho, entonces $H$ también es $\omega$-estrecho.

Demostración. Sea $\pi: G \rightarrow H$ un homomorfismo continuo de un grupo topológico $\omega$-estrecho $G$ sobre un grupo topológico $H$. Sea $V$ una vecindad abierta de la identidad en $H$. El conjunto $U=\pi^{-1}(V)$ es abierto en $G$, por lo tanto, existe un subconjunto numerable $A$ de $G$ tal que $A \cdot U=G$. Vemos que el conjunto $\pi(A)=B$ es numerable y cumple $B \cdot V=\pi(A \cdot U)=\pi(G)=H$. por lo tanto $H$ es $\omega$-estrecho.

Proposición 2.4. El producto topológico de una familia arbitraria de grupos topológicos $\omega$-estrechos es un grupo topológico $\omega$-estrecho.

Demostración. Sea $G=\prod_{i \in I} G_{i}$ un producto cartesiano de grupos $\omega$-estrechos $G_{i}$. Si $U$ es una vecindad abierta de la identidad $e$ en $G$, existe un conjunto abierto canónico $V$ en $G$ tal que $e \in V \subseteq U$. Sean $i_{1}, \ldots, i_{n} \in I$ las coordenadas que satisfacen la igualdad $V=p_{i_{1}}^{-1} p_{i_{1}}(V) \cap \cdots \cap p_{i_{n}}^{-1} p_{i_{n}}(V),(*)$, donde $p_{i}$ es la proyección de $G$ sobre el factor $G_{i}, i \in I$. Notemos que $V_{k}=P_{i_{k}}(V)$ es una vecindad abierta de la identidad en $G_{i_{k}}$ para toda $k \leq n$, escojamos un subconjunto numerable $C_{k}$ de $G_{i_{k}}$ de manera que $C_{k} \cdot V_{k}=G_{i_{k}}$. Definimos el conjunto $C$ de $G$ mediante $C=\prod_{i \in I} A_{i}$, donde $A_{i}=C_{k}$ si $i=i_{k}$ para algún $k \leq n, \mathrm{y} A_{i}=\left\{e_{G_{i}}\right\}$ en caso contrario. Es claro que $|C|=\left|C_{1} \times \cdots \times C_{n}\right| \leq \aleph_{0}$. De (*) se deduce que $C \cdot V=G$, y en consecuencia $C \cdot U=G$. Por lo tanto $G$ es $\omega$-estrecho.

Teorema 2.5. Todo subgrupo $H$ de un grupo topológico $G \omega$-estrecho, es $\omega$-estrecho.

Demostración. Sea $G$ un grupo topológico $\omega$-estrecho y sea $H$ un subgrupo de $G$. Tomemos una vecindad $U$ de $e$ en $H$. Escojamos una vecindad abierta simétrica $V$ de $e$ en $G$ tal que $V^{2} \cap H \subset U$. Dado que $G$ es $\omega$-estrecho, existe un subconjunto numerable $B$ de $G$ tal que $G=B \cdot V$. Sea $C=\{c \in B$ : $c V \cap H \neq \varnothing\}$. Entonces $|C| \leq|B| \leq \omega$, es obvio que $H \subset C V$. Para cada $c \in C$ fijo, $a_{c} \in c V \cap H$, sea $A=\left\{a_{c}: c \in C\right\}$. Como $C$ es numerable, el subconjunto $A$ de $H$ es numerable. afirmamos que $H=A \cdot U$. Como $H$ es un subgrupo de $G$ y $V^{2} \cap H \subset U \subset H$, tenemos que $\left(A V^{2}\right) \cap H \subset A \cdot U \subset H$. Solo nos resta mostrar que $H \subset A \cdot V^{2}$. Claramente, $A \subset H \subset C V$. Dado que que $V$ es simétrica, se sigue que $C \subset A \cdot V$, lo cual implica que $H \subset C V \subset A \cdot V^{2}$. Por lo tanto $H \subset A \cdot U$. Asi $H=A \cdot U$ lo que significa que $H$ es $\omega$-estrecho. 
Proposición 2.6. Todo grupo topológico $G \omega$-estrecho primero numerable es segundo numerable.

Demostración. Sea $\eta=\left\{U_{n}: n \in \omega\right\}$ una base numerable de la identidad $e$ en un grupo topológico $G \omega$-estrecho. Para todo $n \in \omega$ escojamos un conjunto numerable $C_{n} \subset G$ tal que $G=C_{n} \cdot U_{n}$. Entonces la familia $\beta=\left\{x U_{n}: x \in C_{n}, n \in \omega\right\}$ es numerable, afirmamos que $\beta$ es una base para el grupo $G$. En efecto sea $O$ una vecindad del punto $a \in G$. Podemos encontrar $k, l \in \omega$ tal que $a U_{k} \subset O$ y $U_{l}^{-1} U_{l} \subset U_{k}$. Existe $x \in C_{l}$ tal que $a \in x U_{l}$, cuando $x \in a U_{l}^{-1}$. Tenemos $x U_{l} \subset\left(a U_{l}^{-1}\right) U_{l}=a\left(U_{l}^{-1} U_{l}\right) \subset a U_{k} \subset O$, esto es, $x U_{l}$ es una vecindad abierta de $a$ y $x U_{l} \subset O$. Nos resta notar que $x U_{l} \in \beta$.

Teorema 2.7. Todo grupo topológico de Lindelöf es $\omega$-estrecho.

Demostración. Sea $G$ un grupo topológico de Lindelöf y $U$ una vecindad abierta de la identidad e en $G$. La familia $\mathcal{U}=\{x U: x \in G\}$ es una cubierta abierta de $G$. como $G$ es Lindelöf, existe una subfamilia numerable $\mathcal{U}^{\prime}$ de $\mathcal{U}$ que cubre a $G$. Por la definición de $\mathcal{U}$, lo anterior significa que $\mathcal{U}^{\prime}=\{x U: x \in C\}$ cubre a $G$, para un subconjunto numerable $C$ de $G$, es decir $G=C \cdot U$.

Teorema 2.8. Si la celularidad de un grupo topológico $G$ es numerable, entonces $G$ es $\omega$-estrecho.

Demostración. Sea $G$ un grupo topológico de celularidad numerable. Tomemos una vecindad abierta $U$ de la identidad $e$ en $G$. Supongamos que $G \neq C \cdot U$ para todo conjunto numerable $C \subseteq G$. Escojamos una vecindad abierta simétrica $V$ de la identidad $e$ en $G$ tal que $V \cdot V^{-1} \subseteq U$. Recursivamente construimos la sucesión $\left\{x_{\alpha}: \alpha<\omega_{1}\right\}$ de elementos de $G$ de manera que

$$
x_{\beta} \notin x_{\alpha} U \text { para cualquier } \alpha, \beta \text { con } \alpha<\beta<\omega_{1}
$$

Sea $x_{0}=e_{G}$. Si $\gamma<\omega_{1}$ y los elementos $x_{\alpha}$ ya están definidos para $\alpha<\gamma$, podemos escojer $x_{\gamma} \in G \backslash\left(C_{\gamma} \cdot U\right)$, donde $C_{\gamma}=\left\{x_{\gamma}: \alpha<\gamma\right\}$. Claramente, la condición $(*)$ se cumple para todos los $\alpha, \beta \leq \gamma$.

Afirmamos que la familia $\left\{x_{\alpha} V: \alpha<\omega_{1}\right\}$ es celular, es decir, ajena dos a dos. Supongamos lo contrario y escojamos $\alpha, \beta<\omega_{1}$ tales que $\alpha<\beta$ y la intersección $\left(x_{\alpha} V\right) \cap\left(x_{\beta} V\right) \neq \varnothing$. Entonces existen $v_{1}, v_{2} \in V$ tales que $x_{\alpha} v_{1}=x_{\beta} v_{2}$, de donde $x_{\beta} \in x_{\alpha} V \cdot V^{-1} \subseteq x_{\alpha} U$, lo cual contradice $(*)$. 
Observación 2.9. Dado que un espacio separable tiene celularidad numerable, el teorema anterior implica que todo grupo topológico separable es $\omega$-estrecho. Y por lo tanto, el siguente corolario queda establecido.

Corolario 2.10. Todo grupo topológico separable es $\omega$-estrecho.

Como hemos visto la $\omega$-estrechez se hereda a subgrupos. Al contrario, el que un grupo $G$ contenga un subgrupo $\omega$-estrecho no implica que $G$ lo sea. Sin embargo, esta implicación es valida si $H$ es un subgrupo denso en $G$.

Proposición 2.11. Sean $G$ un grupo topológico y $H$ un subgrupo denso de $G$. Si $H$ es $\omega$-estrecho, entonces $G$ también lo es.

Demostración. Sea $U$ cualquier vecindad abierta de la identidad $e$ en $G$. Existe una vecindad abierta y semétrica de $e$ en $G$ tal que $V^{2} \subset U$. Como $H$ es un subgrupo $\omega$-estrecho, existe un conjunto numerable $C \subseteq H$ tal que $H \subseteq C \cdot V$; en particular $C \cdot V$ es denso en $G$. Afirmamos que $G=C \cdot U$. Sea $x \in G$ arbitrario. El conjunto $x V$ es una vecindad de $x$ de modo que $(x V) \cap(C \dot{V}) \neq \varnothing$. Entonces existen $v_{1}, v_{2} \in V$ y $c \in C$ tales que $x v_{1}=c v_{2}$, por lo tanto $x=c v_{2} v_{1}^{-1}=c v_{2} v_{1} \in C \cdot V \cdot V=C \cdot V^{2} \subseteq C \cdot U$. Así $G=C \cdot U$, y por lo tanto $G$ es $\omega$-estrecho. 


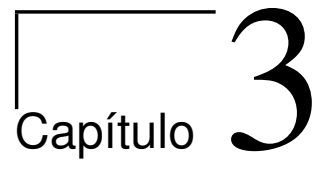

\section{Grupos Topológicos $\omega$-balanceados}

\subsubsection{Definición y Propiedades básicas}

Definición 3.1. Sea $G$ un grupo topológico. Diremos que el número de invariancia de $G$ es menor o igual a $\tau$ donde $\tau$ es un cardinal, en simbolos $\operatorname{Inv}(G) \leq \tau$ si para toda vencidad $U$ de la identidad $e$ en $G$, existe una familia de vecindades abiertas de la identidad $e$ con $|\gamma| \leq \tau$ tal que para toda $x \in G$, podemos encontrar un $V \in \gamma$ que satisface $x V x^{-1} \subset U$.

Decimos que un grupo topológico $G$ es $\omega$-balanceado si $\operatorname{Inv}(G) \leq \omega$ es numerable, esto es si para toda vecindad $U$ de la identidad de $G$, existe una familia numerable $\gamma$ de vencindades de la identidad en $G$ tal que para todo $x \in G$, existe $V \in \gamma$ que satisface $x V x^{-1} \subset U$. Si tal familia $\gamma$ existe se llamará subordinada a $U$.

De la definición anterior, tenemos el siguiente corolario.

Corolario 3.2. Todo subgrupo de un grupo $\omega$-balanceado es también $\omega$ balanceado.

Proposición 3.3. Si $G$ es un grupo topológico $\omega$-estrecho, entonces el número de invariancia de $G$ es numerable, esto es $G$ es $\omega$-balanceado.

Demostración. Sea $U$ una vecindad abierta de la identidad $e$ en $G$. Existe una vecindad abierta y simétrica $V$ de $e$ tal que $V^{3} \subset U$. Como $G$ es $\omega$-estrecho, podemos encontrar un conjunto numerable $A \subseteq G$ tal que $G=V \cdot A$. Entonces para todo $a \in A$, existe una vecidad abierta $W_{a}$ de la identidad $e$ tal que $a W_{a} a^{-1} \subset V$. Afirmamos que la familia $\gamma=\left\{W_{a}: a \in A\right\}$ es la familia que 
estamos buscando. De hecho, $\gamma$ es una familia numerable de vecindades de $e$. Ahora, sea $x \in G$ arbitrario. Entonces $x \in V a$, para toda $a \in A$, y por lo tanto, $x W_{a} x^{-1} \subset V a W_{a} a^{-1} V^{-1} \subset V \cdot V \cdot V^{-1} \subset V^{3} \subset U$, esto es, $\gamma$ es subordinada a $U$.

Teorema 3.4. El número de invariancia de un grupo topológico arbitrario $G$ primero numerable es numerable.

Demostración. Sea $\left\{V_{n}: n \in \omega\right\}$ una base numerable del espacio $G$ en la identidad $e$ de $G$. Tomemos cualquier vecindad abierta $U$ de $e$. Entonces $U x$ y $x U$ son vecindades abiertas de $x$. Como la traslaciónes izquierda y derecha de $x$ son continuas y $x e \in U x$, ex $\in x U$, existe $n \in \omega$ tal que $x V_{n} \subset U x$ y $V_{n} x \subset x U$. De esto tenemos que $x V_{n} x^{-1} \subset U x x^{-1}=U$ y $x^{-1} V_{n} x \subset x^{-1} x U=U$. Por lo tanto, $\operatorname{Inv}(G) \leq \omega$

Corolario 3.5. Todo grupo topológico metrizable es $\omega$-balanceado.

Lema 3.6. Sea $G$ es un grupo topológico $\omega$-balanceado,y sea $\gamma$ una familia numerable de vecindades abiertas de la identidad e en $G$. Entonces existe una familia numerable $\gamma^{*}$ de vecindades abiertas de e con las siguientes propiedades:

1) $\gamma \subset \gamma^{*}$

2) La intersección de cualquier subfamilia finita de $\gamma^{*}$ esta en $\gamma^{*}$;

3) Para cada $U \in \gamma^{*}$, existe una vecindad simétrica $V \in \gamma^{*}$ tal que $V^{2} \subset U$

4) Para cada $U \in \gamma^{*}$ y todo $a \in G$, existe $V \in \gamma^{*}$ tal que $a V a^{-1} \subset U$.

Demostración. Sea $G$ un grupo topológico $\omega$-balanceado, y sea $\gamma$ una familia numerable de vecindades abiertas de e en $G$. Para cada $U \in \gamma$, fijemos una vecindad abierta simétrica $V_{U}$ de $e$ tal que $V_{U}^{2} \subset U$. Fijemos también una familia numerable $\mathcal{V}_{U}$ de vecindades abiertas de $e$ subordinada a $U$. Ahora sea

$$
\phi(\gamma)=\{\cap \lambda: \lambda \subset \gamma,|\lambda|<\omega\} \cup \bigcup\left\{\mathcal{V}_{U}: U \in \gamma\right\} \cup\left\{V_{U}: U \in \gamma\right\} .
$$

Sea $\gamma_{0}=\gamma, \gamma_{1}=\phi\left(\gamma_{0}\right)$ y repetimos está operación, definimos por inducción las familias numerables $\gamma_{2}, \gamma_{3}$ y por la regla $\gamma_{n+1}=\phi\left(\gamma_{n}\right)$, para todo $n \in \omega$. Sea $\gamma^{*}=\bigcup_{n \in \omega} \gamma_{n}$. Dado que $\gamma_{n} \subset \gamma_{n+1}$ para todo $n \in \omega$, y vemos de la definición de arriba, que la familia $\gamma^{*}$ satisface las condiciones $(1)-(4)$. 
Lema 3.7. Sean $G$ es un grupo topológico w-balanceado, y $U$ una vecindad abierta de la identidad e en $G$. Entonces existe una sucesión $\left\{U_{n}: n \in \omega\right\}$ de vecindades abiertas de e tal que, para cada $n \in \omega$, las siguientes condiciones se cumplen:

a) $U_{0} \subset U$;

b) $U_{n}^{-1}=U_{n}$;

c) $U_{n+1}^{2} \subset U_{n}$;

d) Para cada $x \in G$ y cada $n \in \omega$, existe $k \in \omega$ tal que $x U_{k} x^{-1} \subset U_{n}$.

Demostración. Sea $\gamma=\{U\}$, y tomemos una familia numerable $\gamma^{*}$ de vecindades abiertas de la identidad $e$ que satisface las condiciones (1) - (4) del lema 3.6. Entonces $U \in \gamma *$. Definiremos, por inducción, una sucesión de elementos de $\gamma *$. Primero enumeraremos los elementos de $\gamma^{*}$, diremos, $\gamma^{*}=\left\{W_{n}: n \in \omega\right\}$. Escojamos $U_{0}$ cualquier elemento simétrico de $\gamma^{*}$ tal que $U_{0} \subset U \cap W_{0}$. Dado que $\gamma^{*}$ satisface la condiciones (2) y (3), podemos tomar un elemento simétrico $V$ de $\gamma^{*}$ tal que $V^{2} \subset U_{n} \cap \bigcap_{i=0}^{n} W_{i}$.

Sea $U_{n+1}=V$. Por lo tanto la definición de está sucesión esta completa.

Es inmediato de la construcción de la sucesión $\left\{U_{n}: n \in \omega\right\}$ que satisface las condiciones de la $(a)-(c)$. Mostraremos ahora que la condición $(d)$ también se cumple. Fijemos $n \in \omega$ y $x \in G$. Como la familia $\gamma^{*}$ cumple la condición (4) del lema 3.6, existe $j \in \omega$ tal que $x W_{j} x^{-1} \subset U_{n}$. Tomando $k=\operatorname{máx}\{n, j\}$. Tenemos entonces que $U_{k+1} \subset U_{k+1}^{2} \subset W_{j}$, por la definición inductiva de $U_{k+1}$. Por lo tanto $x U_{k+1} x^{-1} \subset x W_{j} x^{-1} \subset U_{n}$. Por lo tanto la condición $(d)$ se cumple y el lema queda completamente probado.

Teorema 3.8. Sea $G$ un grupo topológico w-balanceado. Entonces para toda vecindad abierta $U$ de la identidad e en $G$, existe una pseudométrica continua izquierdo-invariante $\rho$ sobre $G$ tal que las siguientes condiciones se cumplen:

(p1) $\{x \in G: \rho(e, x)<1\} \subset U$;

(p2) $\{x \in G: \rho(e, x)=0\}$ es un subgrupo cerrado invariante de $G$;

(p3) para todos $x, y \in G, \rho(e, x y) \leq \rho(e, x)+\rho(e, y)$.

Demostración. Por el lema 3.7, podemos encontrar una sucesión $\left\{U_{n}: n \in \omega\right\}$ de vecindades abiertas de $e$ en $G$ que satisface las condiciones $(a)-(d)$ del lema 3.7. Por el lema 3.6 y el lema 1.36, existe una prenorma continua $N$ sobre $G$ tal que la siguiente condición se cumple: 
(PN4) $\left\{x \in G: N(x)<1 / 2^{n}\right\} \subset U_{n} \subset\left\{x \in G: N(x) \leq 2 / 2^{n}\right\}$.

Ahora, para $x, y \in G$ arbitrarios, sea $\rho(x, y)=N\left(x^{-1} y\right)$. Entonces la continuidad de $N$ implica que $\rho$ también es continua. Es claro de 3.0.2 y la condición $(a)$ del lema 3.8 que $(p 1)$ se cumple.

Afirmación 1: $\rho$ es una pseudométrica sobre el conjunto $G$.

De hecho para todos $x, y$ en $G$ tenemos: $\rho(x, y)=N\left(x^{-1} y\right) \geq 0$ y $\rho(y, x)=$ $N\left(y^{-1} x\right)=N\left(\left(y^{-1} x\right)^{-1}\right)=N\left(x^{-1} y\right)=\rho(x, y)$. También $\rho(x, x)=N\left(x^{-1} x\right)=$ $N(e)=0$. Además, para todos $x, y, z$ en $G$, tenemos:

$$
\begin{aligned}
\rho(x, z) & =N\left(x^{-1} z\right)=N\left(x^{-1} y y^{-1} z\right) \leq N\left(x^{-1} y\right)+N\left(y^{-1} z\right) \\
& =\rho(x, y)+\rho(y, z)
\end{aligned}
$$

Por lo tanto $\rho$ satisface la desigualdad triangular.

Afirmación 2: La pseudométrica $\rho$ es izquierdo-invariante.

De hecho, $\rho(z x, z y)=N\left((z x)^{-1} z y\right)=N\left(x^{-1} z^{-1} z y\right)=N\left(x^{-1} y\right)=\rho(x, y)$, para $x, y, z$ en $G$ arbitrarios.

Hagamos $Z=\{x \in G: N(x)=0\}$. Notemos que $\rho(e, x)=N(x)$, para cada $x \in G$, ya que $\rho(e, x)=N\left(e^{-1} x\right)=N(x)$. Por lo tanto, tenemos que $Z=\{x \in G: \rho(e, x)=0\}$.

Afirmación 3: $Z=\bigcap_{n \in \omega} U_{n}$.

Esto se sigue claramente de la condición (PN4).

Afirmación 4: $Z$ es un subgrupo cerrado invariante de $G$.

Dado que la prenorma $N$ es continua, el conjunto $Z$ es cerrado en el espacio $G$. Y por la proposición 1.29 tenemos que $Z$ es un subgrupo de $G$.

Sólo nos resta probar que el subgrupo $Z$ de $G$ es invariante. Tomemos cualquier $x \in G$. Debemos ver que $x Z x^{-1} \subset Z$. Vemos por la Afirmación 3 que es suficiente probar que $x Z x^{-1} \subset U_{n}$, para cada $n \in \omega$. Fijemos un $n \in \omega$. De la condición $(d)$ del lema 3.7 tenemos que existe $k \in \omega$ tal que $x U_{k} x^{-1} \subset U_{n}$. Como $Z \subset U_{k}$, concluimos que $x Z x^{-1} \subset U_{n}$, esto es, $Z$ es invariante.

La condición $(p 3)$ se cumple trivialmente, dado que $N$ es una prenorma y $\rho(e, x)=N(x)$.

Necesitamos los siguientes dos lemas sencillos.

Lema 3.9. Si $N$ es una prenorma sobre un grupo $G$, y z es un elemento de $G$ tal que $N(z)=0$, entonces $N(z x)=N(x z)$, para cada $x \in G$. 
Demostración. Tenemos que $N(z x) \leq N(z)+N(x)=N(x)$. De manera similar, $N(x)=N\left(z^{-1} z x\right) \leq N\left(z^{-1}\right)+N(z x) \leq N(z x)$, dado que $N\left(z^{-1}\right)=$ $N(z)=0$. Por lo tanto, $N(z x)=N(x)$. De manera analoga se sigue que $N(x)=N(x z)$.

Lema 3.10. En la notación de la prueba del teorema 3.8, si a y b son elementos cualesquieras de $G$, y si $a_{1} \in a Z, b_{1} \in b Z$. Entonces $\rho\left(a_{1}, b_{1}\right)=$ $\rho(a, b)$.

Demostración. Supongamos que $b=b_{1}$. Como $a_{1} \in a Z$, entonces $a_{1}=a z$, para algún $z \in Z$. Entonces usando $N\left(z^{-1}\right)=N(z)=0$ y el lema 3.9. obtenemos $\rho\left(a_{1}, b\right)=N\left((a z)^{-1} b\right)=N\left(z^{-1} a^{-1} b\right)=N\left(a^{-1} b\right)=\rho(a, b)$.

Continuaremos utilizando los objetos construidos en la demostración del teorema 3.8, en particular, fijaremos la métrica $\rho$ en $G$ construida anteriormente. Sean $H=G / Z$ el grupo cociente, y $\pi$ el homomorfismo canónico de $G$ sobre $H$. Sean $A, B \in H$ elementos cualesquiera. Escojamos cualquieras $a \in A \mathrm{y}$ $b \in B$, y sea $d(A, B)=\rho(a, b)$. Por el lema 3.10, la definición de $d(A, B)$ no depende de la elección de $a \in A$ y $b \in B$.

Ahora definamos la función $N_{H}$ en $H$ por la regla $N_{H}(A)=N(a)$ para toda $A \in H$ y $a \in A$. por el lema $3.9 N$ no depende de la elección de $a \in A$.

Con estas definiciones tenemos que $d(\pi(a), \pi(b))=\rho(a, b)$ para cualquier $a, b \in G$, y $N_{H}(\pi(a))=N(a)$, para cualquier $a \in G$.

Dado que $\pi$ es un homomorfismo de $G$ sobre $H$, y $\pi(Z)$ es el elemento neutro $E$ del grupo $H$, se sigue de la definición de $Z$ y $\rho$ que $d$ es una métrica sobre $H$ y $N_{H}$ es una prenorma sobre $H$ que cumple la siguiente condición adicional:

(PN5) Si $N_{H}(A)=0$, entonces $A$ es el elemento neutro $E$ de $H$.

Ahora para todo $\varepsilon>0$, sean $B(\varepsilon)=\{x \in G: N(x)<\varepsilon\}$ y $O(\varepsilon)=\{X \in H$ : $\left.N_{H}(X)<\varepsilon\right\}$. Claramente $\pi(B(\varepsilon))=O(\varepsilon)$, para todo $\varepsilon>0$. Notemos que la prenorma $N$ también satisface la siguiente condición:

(PN6) Para todo $\varepsilon>0$ y todo $x \in G$, existe un $\delta>0$ tal que $x B(\delta) x^{-1} \subset B(\varepsilon)$.

Dado que $\pi(B(\varepsilon))=O(\varepsilon)$, se sigue que para la métrica $d$ sobre $H$ tenemos:

(d1) Para todo $\varepsilon>0$ y todo $X \in H$, existe $\delta>0$ tal que $X O(\delta) X^{-1} \subset O(\varepsilon)$. Dado que $\rho(e, x)=N(x)=N\left(x^{-1}\right)=\rho\left(e, x^{-1}\right)$, de la definición de $d$ se sigue que: 
(d2) $d(E, X)=d\left(E, X^{-1}\right)$, para todo $X \in H$.

Se sigue de $(d 2)$ que $O(\varepsilon)=(O(\varepsilon))^{-1}$, para todo $\epsilon>0$.

También de $(p 3)$ y de la definición de $d$, obtenemos:

(d3) $\left(O\left(1 / 2^{n+1}\right)\right)^{2} \subset O\left(1 / 2^{n}\right)$.

Usando (PN5), concluimos que

(d4) $\{E\}=\bigcap_{n \in \omega} O\left(1 / 2^{n}\right)$.

Ahora sea $\tau^{*}$ la topología generada por la métrica $d$ en $H$. Vamos a demostrar que $H$ con esta topología es un grupo topológico. De hecho, dado que $d$ es izquierdo-invariante es suficiente observar que la familia $\left\{O\left(1 / 2^{n}\right): n \in \omega\right\}$, la cual es una base del espacio en el elemento neutro $E$, satisface los axiomas de base de grupo topológico en el elemento neutro. Y esto es exactamente que las condiciones $(d 1)-(d 4)$ se garantizan. Por lo tanto, $H$ con la topología $\tau^{*}$ es un grupo topológico.

Finalmente, la igualdad $\pi(B(\varepsilon))=O(\varepsilon)$, donde $\varepsilon>0$, implica que el homomorfismo $\pi$ de $G$ sobre $H=G / Z$ es continuo en el elemento neutro. Dado que $G$ y $H$ son grupos topológicos, se sigue que $\pi$ es continuo. Notemos también que si $x \in G, \pi(x)=X$, y $\varepsilon>0$, entonces $N(x)<\varepsilon$ es equivalente a $N_{H}(X)<\epsilon$.

Por lo tanto, $\pi^{-1}(O(\varepsilon))=B(\varepsilon)$ para todo $\varepsilon>0$. En particular, $\pi^{(-1 O(1))}=$ $B(1) \subset U$.

Por lo tanto con lo anterior el siguiente teorema queda establecido, y sugiere la idea de encajar un grupo $\omega$-balanceado en producto de grupos metrizables.

Teorema 3.11. Si $G$ es un grupo $\omega$-balanceado, entonces para toda vecindad abierta $U$ de la identidad e en $G$, existe un homomorfismo continuo $\pi$ de $G$ sobre un grupo metrizable $H$ tal que $\pi^{-1}(V) \subset U$, para alguna vecindad $V$ de la identidad $e^{*}$ en $H$.

El teorema anterior tiene un corolario importante.

Corolario 3.12. Si $G$ es un grupo $\omega$-estrecho, entonces para toda vecindad abierta $U$ de la identidad e en $G$, existe un homomorfismo continuo $\pi$ de $G$ sobre un grupo topológico segundo numerable $H$ tal que $\pi^{-1}(V) \subset U$, para alguna vecindad $V$ de la identidad $e^{*}$ en $H$.

Demostración. Por el teorema 3.11, podemos encontrar un homomorfismo continuo $\pi$ de $G$ sobre un grupo topológico metrizable $H$ y una vecindad 
abierta $V$ de la identidad $e^{*}$ en $H$ tal que $\pi^{-1}(V) \subset U$. de la proposición 2.3 , tenemos que el grupo $H$ es $\omega$-estrecho, por lo tanto la proposición 2.6 implica que $H$ es segundo numerable.

Definición 3.13. Decimos que un grupo topológico $G$ es de rango-metrizable si para toda vecindad abierta $U$ de la identidad $e$ en $G$, existe un homomorfismo continuo $\pi$ sobre un grupo metrizable $H$ tal que $\pi^{-1}(V) \subset U$, para alguna vecindad abierta $V$ de la identidad $e^{*}$ de $H$.

Observación 3.14. Claramente el teorema 3.11lo podemos reformular como sigue: Si $G$ es un grupo topológico $\omega$-balanceado, entonces $G$ es de rangometrizable.

Definición 3.15. Sea $\mathcal{P}$ cualquier clase de grupos topológicos, y sea $G$ cualquier grupo topológico. Decimos que $G$ es de rango $-\mathcal{P}$ si para toda vecindad abierta $U$ de la identidad $e$ en $G$, existe un homomorfismo continuo $\pi$ de $G$ en un grupo $H \in \mathcal{P}$ tal que $\pi^{-1}(V) \subset U$, para alguna vecindad abierta $V$ de la identidad $e^{*}$ en $H$.

Observación 3.16. De la definición anterior se sigue inmediatamente que todo subgrupo $H$ de un grupo $G$ de rango $-\mathcal{P}$ es también de rango $-\mathcal{P}$. Similarmente, el corolario 3.12 es equivalente a decir que todo grupo $\omega$ estrecho es de rango $-\Omega$, donde $\Omega$ es la clase de los grupos topológicos segundo numerable

El siguiente hecho se desprende de la definición de la topología del producto.

Proposición 3.17. Sea P cualquier clase de grupos topológicos cerrada bajo productos finitos, y sea $H$ el producto topológico de la familia $\left\{H_{a}: a \in A\right\}$ de grupos en la clase $\mathcal{P}$. Entonces todo subgrupo de $H$ es de rango $-\mathcal{P}$.

Teorema 3.18. Sea $\mathcal{P}$ una clase de grupos topológicos, $\tau$ un cardinal infinito, y $G$ un grupo topológico que es rango $-\mathcal{P}$ y tiene una base $\mathcal{B}$ de vecindades abiertas del elemento neutro tal que $|\mathcal{B}| \leq \tau$. Entoces $G$ es topológicamente isomorfo a un subgrupo del producto de una familia $\left\{H_{a}: a \in A\right\}$ de grupos tal que $H_{a} \in \mathcal{P}$, para todo $a \in A$ y $|A| \leq \tau$.

Demostración. Fijemos una base $\mathcal{B}$ de vecindades abiertas de la identidad $e$ en $G$ tal que $|\mathcal{B}| \leq \tau$. Así, como $G$ es de rango $-\mathcal{P}$, para todo $U \in \mathcal{B}$ existe un homomorfismo continuo $\varphi_{U}$ de $G$ en un grupo $H_{U} \in \mathcal{P}$ tal que 
$\left(\varphi_{U}\right)^{-1}(V) \subset U$, para alguna vecindad abierta $V$ de la identidad $e^{*}$ en $H$. Definamos a $\varphi$ como el producto diagonal de la familia $\left\{\varphi_{U}: U \in \mathcal{B}\right\}$. Afirmamos que $\varphi$ es un isomorfismo topológico de $G$ sobre un subgrupo del producto topológico de la familia $\left\{H_{U}: U \in \mathcal{B}\right\}$. En efecto, si probamos que la función $\varphi$ es inyectiva y que además separa puntos de cerrados, habremos probado que $\varphi$ es un isomorfismo topológico. Veamos que $\varphi$ es inyectiva o, equivalentemente, que $\operatorname{ker} \varphi$ consta sólo del elemento $e$. Sea $g \in G$ con $g \neq e$ escojamos $U \in \mathcal{B}$ tal que $g \notin U$. Sea $p_{U}$ la proyección del producto de la familia $\left\{H_{U}: U \in \mathcal{B}\right\}$ al factor $H_{U}$. Consideremos la vecindad $W=p_{U}^{-1}(V)$ de la identidad en el producto de la familia $\left\{H_{U}: U \in \mathcal{B}\right\}$. De la igualdad $\varphi_{U}=p_{U} \circ \varphi$ se tiene que $\varphi^{-1}(W)=\varphi_{U}^{-1}(V) \subseteq U$. Notemos que $\varphi(g) \notin W$, es decir, $\varphi(g)$ es distinto de la identidad en el producto de la familia $\left\{H_{U}: U \in \mathcal{B}\right\}$, esto es, que $\operatorname{ker} \varphi$ consta solamente de la identidad. Por otro lado, si tomamos la familia $\mathcal{F}=\left\{\varphi_{U}: U \in \mathcal{B}\right\}$ y probamos que separa puntos de cerrados, la demostración está completa. Sean $e$, la identidad del grupo $G, C$ un cerrado tal que $e \notin C$ y $U \in \mathcal{B}$. Entonces $e \in U \subset G \backslash C$. Ahora, si $\varphi_{U}: G \rightarrow H_{U}$ y $V \in H$ son tales que $\varphi_{U}^{-1}(V) \subset U \subset G \backslash C$, entonces $\varphi_{U}(e) \in V$. Por demostrar que $V \cap \varphi_{U}(C)=\varnothing$. En efecto, sea $v \in V$. Entonces $\varphi_{U}^{-1}(v) \subset \varphi^{-1}(V) \subset U \subset G \backslash C$ y así $v \in V \subset \varphi(G \backslash C)=$ $\varphi(G) \backslash \varphi(C)$, por lo tanto, $v \notin \overline{\varphi(C)}$. Así, la familia $\mathcal{F}$ separa puntos de cerrados lo cual implica que $\varphi$ es un encaje homeomórfico.

Teorema 3.19 (G. I. Katz. 1953). Para todo grupo topológico G las siguientes tres condiciones son equivalentes:

1) $\operatorname{Inv}(G) \leq \omega$;

2) G es rango - metrizable;

3) G es topológicamente isomorfo a un subgrupo de un producto topológico de grupos metrizables.

Demostración. De la proposición 3.17 y del teorema 3.18 tenemos que 2) y 3) son equivalentes. Por el teorema 3.11 tenemos que 1) y 2) son equivalentes. Sólo nos resta mostrar que 2) y 1) son equivalentes, tomemos una vecindad abierta $U$ de la identidad $e$ en $G$ en un grupo $G$ de rango - metrizable y consideremos un homomorfismo continuo $\pi: G \rightarrow H$ de $G$ sobre un grupo metrizable $H$ tal que $\pi^{-1}(V) \subset U$ para alguna vecindad abierta $V$ de la identidad $e^{*}$ en $H$. Ahora sea $\mathcal{B}$ una base numerable de la identidad $e^{*}$ en $H$. 
Por lo tanto la familia numerable $\left\{\pi^{-1}(O): O \in \mathcal{B}\right\}$ de vecindades abiertas de la identidad $e$ en $G$ esta subordinada a $U$, en conclusión $\operatorname{Inv}(G) \leq \omega$.

Teorema 3.20 (I. I. Guran. 1981). Un grupo topológico $G$ es w-estrecho si y sólo si $G$ es topológicamente isomorfo a un subgrupo de un producto cartesiano de grupos segundo numerables.

Demostración. " $\Rightarrow$ "

Sea $\Omega$ la clase de los grupos topológicos segundo-numerables. Por el corolario 3.12, todo grupo $\omega$-estrecho es de rango $-\Omega$, y por el teorema 3.18 tenemos que $G$ es topológicamente isomorfo a un subgrupo del producto de grupos de la familia $\Omega$.

$" \Leftarrow "$

Por la proposición 2.4 y el teorema 2.5, tenemos que todo subgrupo de un producto topológico de grupos topológicos segundo-numerable es $\omega$-estrecho.

Finalmente daremos un ejemplo de un grupo topológico que no es $\omega$ balanceado.

Ejemplo 3.21. Sea $X$ el espacio de dos flechas es decir $X=C_{0} \cup C_{1} \subset \mathbb{R}^{2}$, donde $C_{0}=\{(x, 0): 0<x \leq 1\}$ y $C_{1}=\{(x, 1): 0 \leq x<1\}$, y la topología sobre $X$ está generada por la base que consiste de los conjuntos de la forma

$$
\left\{(x, i) \in X: x_{0}-1 / k<x<x_{0} i=0,1\right\} \cup\{(x, 0)\},
$$

donde $0<x_{0} \leq 1$ y $k=1,2, \ldots$, y los conjuntos de la forma

$$
\left\{(x, i) \in X: x_{0}<x<x_{0}+1 / k i=0,1\right\} \cup\{(x, 1)\},
$$

donde $0 \leq x_{0}<1$ y $k=1,2, \ldots$.

Sea $G=H o m e o(X)$ el grupo de todos los homeomorfismos de $X$ sobre si mismo, con la topología compacto-abierta, es decir la topología $\tau(X, X)=\mathcal{T}$ generada por la base que consiste de todos los conjuntos $\bigcap_{i=1}^{k} M\left(C_{i}, U_{i}\right)$, donde $C_{i}$ es un subconjunto compacto de $X$ y $U_{i}$ es un subconjunto abierto de $X$ para $i=1,2, \ldots, k$.. Entonces $(G, \mathcal{T})$ es un grupo topológico el cual no es $\omega$-balanceado.

Solución. Consideremos a $C_{0}$ como un subespacio de $X$, entonces, para cualquier $z=(t, 0) \in C_{0}$, la familia $\{(a, t] \times\{0\}: 0<a<t\}$ es una base del 
punto $z$. Una fácil consecuencia es que la función $i: C_{0} \rightarrow[-1,0)$ definida por $i(t, 0)=-t$ es un homeomorfismo si consideramos a $[-1,0)$ como un subespacio de la recta de Sorgenfrey $S$.

De la misma forma, si consideramos a $C_{1}$ como un subespacio de $X$, entonces para cualquier $z=(t, 1) \in C_{1}$, la familia $\{[t, a) \times\{1\}: t<a<1\}$ es una base del punto $z$, por lo tanto es inmediato que la función $i: C_{1} \rightarrow[0,1)$ definida por $i(t, 1)=t$ es un homeomorfismo si consideramos a $[0,1)$ como un subespacio de la recta de Sorgenfrey $S$.

Vamos a probar ahora que el espacio $X$ es Hausdorff y compacto. Consideremos la función $\pi: X \rightarrow[0,1]$ definida por $\pi(t, i)=t$ esta función es continua si consideramos a $[0,1]$ como un subespacio con la topología inducida de $\mathbb{R}$. Tomemos cualquier $z_{0}, z_{1} \in X$ con $z_{0} \neq z_{1}$. Si $\pi\left(z_{0}\right) \neq \pi\left(z_{1}\right)$, entonces existen conjuntos $U_{i} \in \tau\left(\pi\left(z_{i}\right),[0,1]\right), i=0,1$ tal que $U_{0} \cap U_{1}=\varnothing$. Entonces $V_{0}=\pi^{-1}\left(U_{0}\right)$ y $\pi^{-1}\left(U_{1}\right)$ son abiertos en $X$ y separa a los puntos $z_{0} \mathrm{y} z_{1}$. Ahora si $z_{0}=(t, 0)$ y $z_{1}=(t, 1)$ para algún $t \in(0,1)$, entonces consideremos los conjuntos $U_{0}=((0, t] \times\{o\}) \cup((0, t) \times\{1\})$ y $U_{1}=([t, 1) \times\{1\}) \cup((t, 1) \times\{0\})$. Es inmediato que $U_{i} \in \tau\left(z_{i}, X\right)$ y $U_{0} \cap U_{1}=\varnothing$, por lo tanto el espacio $X$ es Hausdorff.

Para demostrar que $X$ es compacto primero mostraremos que $\pi$ es cerrada. Es claro que la función $\pi$ es sobreyectiva por lo tanto para probar que $\pi$ es cerrada usaremos el siguiente hecho. Una función continua y sobreyectiva $f: Y \rightarrow Z$ es cerrada si y sólo si para cualquier $z \in Z$ y cualquier $O \in \tau\left(f^{-1}(z), Y\right)$, existe $O^{\prime} \in \tau(z, Z)$ tal que $f^{-1}\left(O^{\prime}\right) \subset O$. Tomemos un punto cualquiera $t \in(0,1)$ y un conjunto cualquiera $W \in \tau\left(\pi^{-1}(t), X\right)$; si $z_{i}=(t, i)$ para $i \in\{0,1\}$ entonces $\pi^{-1}(t)=\left\{z_{0}, z_{1}\right\}$. Como $z_{0} \in W$, existe $a \in(0, t)$ tal que $U_{0}=((a, t] \times\{0\}) \cup((a, t) \times\{1\}) \subset W$. De la misma forma si $z_{1} \in W$, existe $b \in(t, 1)$ tal que $U_{1}=([t, b) \times\{1\}) \cup((t, b) \times\{0\}) \subset W$. Ahora, el conjunto $V=(a, b)$ es un abierto en $[0,1]$; por otra parte tenemos que $t \in V$ y $\pi^{-1}(V) \subset W$. Por lo tanto, para todo $t \in(0,1)$ y todo $W \in \tau\left(\pi^{-1}(t), X\right)$ existe $V \in \tau(t,[0,1])$ tal que $\pi^{-1}(V) \subset W$. La prueba de la misma propiedad en los puntos $t=0$ y $t=1$ es fácil por lo tanto la omitiremos. Así podemos concluir que la función $\pi$ es cerrada. El conjunto $\pi^{-1}(z)$ consiste a lo mas de dos puntos para cada $z \in X$, por lo tanto $\pi^{-1}(z)$ es compacto para todo $z \in X$. Así como la función $\pi$ es continua, sobreyectiva, cerrada y tiene fibras compactas, podemos concluir que $\pi$ es una función perfecta; ahora como [0,1] es compacto, concluimos que $X$ es compacto. Es claro que el espacio $X$ es cero-dimensional homogéneo y primero numerable. Además $X$ no es metrizable, por lo tanto $(G, \mathcal{T})$ es un grupo topológico 
que no es $\omega$-balanceado. Ya vimos que $X$ es un espacio cero-dimensional homogéneo, primero numerable Hausdorff y compacto. Si $G$ fuera $\omega$-balanceado la compacidad de $X$ implicaria que $X$ es metrizable por el colorario A.39 
$\prod_{\text {Capítulo }}$

\section{Conclusiones y futuras Direcciones}

Tratar de caracterizar los grupos topológicos, en general, es una tarea muy ambiciosa. En este trabajo tratamos de dar un estudio detallado de dos clases, muy importantes, de grupos topológicos: Los $\omega$-estrechos y los $\omega$-balanceados. Uno de nuestros objetivos fue estudiar detalladamente las propiedades de los grupos $\omega$-estrechos. A estudiar esta clase de grupos pudimos ver que se comportaban muy bien bajo las operaciones más frecuentes en grupos. Observamos que esta clase se conserva bajo imágenes através de homomorfismos continuos, además, era cerrada bajo productos arbitrarios y bajo subgrupos, como lo muestran más detalladamente las proposiciones 2.3. 2.4 y 2.5 .

Observamos también que si un grupo topológico $G$ tiene celularidad numerable, entonces es $\omega$-estrecho; además, pudimos notar que el tener un subgrupo $H \omega$-estrecho de un grupo topológico $G$ no implica que el grupo $G$ sea $\omega$ estrecho, a menos que el subgrupo $H$ sea denso en $G$.

Siguiendo este desarollo, logramos introducir otra clase muy importante de grupos topológicos, los $\omega$-balanceados. En esta clase de grupos pudimos notar que se conservaban sus propiedades bajo la operación de tomar subgrupos; además, pudimos obsevar que todo grupo $\omega$-estrecho era $\omega$-balanceado.

Así pudimos, después de trabajar sobre estas dos clases de grupos topológicos, dar caracterizaciones sobre ellos, como lo muestra el Teorema de Guran 3.20 y el Teorema de Kats 3.19. Estos dos teoremas nos dicen que un grupo topológico es $\omega$-estrecho, respectivamente, $\omega$-balanceado si y sólo si se puede encajar como un subgrupo de un producto de grupos segundo-numerable, respectivamente, primero-numerable. 
Estos teoremas fueron la piedra angular de nuestro trabajo.

Una continuación natural en el estudio de los grupos $\omega$-estrechos y $\omega$ balanceados es el estudio de los grupos $\mathbb{R}$-factorizables. Es por eso que éste tema constituirá nuestro futuro trabajo de investigación; nos apoyaremos en la teoría y técnicas aprendidas en la realización de esta tesis para estudiar la estabilidad de esta nueva clase de grupos topológicos.

Otra futura linea de investigación seria tratar de aplicar la teoría de los grupos $\omega$-estrechos y $\omega$-balanceados a una clase más débil de grupos como los grupos paratopológicos, semitopológicos, etc. 
$\Gamma_{\text {Apéndice }} \mathrm{A}$

\section{Acciones de Grupos Topológicos sobre Espacios Topológicos}

En este apartado, introducimos conceptos básicos y resultados elementales de acciones de grupos topológicos sobre espacios topológicos. Definiremos algunos conceptos importantes como acciones de grupos, espacios de Dugundji, funciones de suavidad-cero, conexión, etc., y presentaremos algunos teoremas de acciones de grupos topológicos sobre espacios topológicos. Todas la demostraciones se omiten. En cambio, el lector encotrará la referencia donde se demuestran los resultados mencionados. Todos los resultados se encuentran en 4 .

\section{A.1. Espacios Dugundji y funciones de suavidad- cero}

Definición A.1. Un espacio compacto $X$ es llamado Dugundji si para todo espacio compacto cero-dimensional $Z$ y toda función continua $f: A \rightarrow X$, donde $A$ es un subconjunto cerrado de $Z$, existe una función $g: Z \rightarrow X$ que extiende a $f$.

La siguiente proposición establece dos propiedades básicas de los espacios de Dugudji.

Proposición A.2. La clase de los espacios de Dugundji tiene las siguientes propiedades: 
a) todo espacio compacto metrizable es Dugundji;

b) el producto de una familia arbitraria de espacios Dugundji is Dugundji.

Combinando $a$ ) y b) en la proposición anterior, deducimos lo siguiente:

Corolario A.3. El producto de una familia arbitraria de espacios compactos segundo-numerables es Dugundji.

Vamos, ahora, a establecer un hecho simple pero importante.

Teorema A.4 (R. Haydon). Todo espacio Dugundji es diádico.

Definición A.5. Una función continua $f: X \rightarrow Y$ es llamada de suavidadcero si para todo espacio compacto cero-dimensional $Z$, toda función continua $g: Z \rightarrow Y$ y una función continua $h: A \rightarrow X$ de un subconjunto cerrado $A$ de $Z$ que satisface $g\lceil A=f \circ h$, existe una función continua $\varphi: Z \rightarrow X$ que extiende a $h$

Proposición A.6. Si $f: X \rightarrow Y$ es una función de suavidad-cero entre espacios compactos $X$ y $Y$, entonces $f(X)=Y$.

Una de las propiedades más importantes de las funciones de suavidad-cero es la siguiente.

Proposición A.7. La composición de funciones de suavidad-cero entre espacios compactos es una función de suavidad-cero.

Definición A.8. Una función continua $f: X \rightarrow Y$ tiene núcleo metrizable si existe un espacio métrico compacto $C$ y un encaje topológico $i: X \rightarrow Y \times C$ tal que $f=p \circ i$, donde $p: Y \times C \rightarrow Y$ es la proyección.

Resulta que las funciones continuas abiertas con núcleo metrizable son de suavidad-cero.

Teorema A.9. Sea $f: X \rightarrow Y$ una función continua, abierta y sobreyectiva de un espacio compacto. Si $f$ tiene núcleo metrizable, entonces $f$ es de suavidad-cero.

Definición A.10. Una función continua es llamada casi abierta si, para todo subconjunto abierto $U \subset X$, existe un conjunto abierto $V \subset Y$ tal que $f(U)$ es un subconjunto denso de $V$. Evidentemente, $f$ es casi abierta si y sólo si $f(U) \subset \operatorname{Int}_{y} c l_{y}(f(U))$, para todo conjunto abierto $U \subset X$. Toda función abierta es casi abierta, pero no viceversa. 
Proposición A.11. Supongamos que $f: X \rightarrow Y$ es una función continua abierta y $S$ es un subespacio denso de $X$. Entonces la función $f\lceil S: S \rightarrow Y$ es casi abierta.

Corolario A.12. Si $S$ es un subespacio denso de un espacio producto $X \times Y$. Entonces la restricción a $S$ de la proyección $p: X \times Y \rightarrow X$ es una función casi abierta de $S$ a $Y$.

Lema A.13. Las siguientes condiciones son equivalentes para una función continua $f: X \rightarrow Y$

a) $f$ es casi abierta;

b) $\overline{f^{-1}(O)}=f^{-1}(\bar{O})$ se cumple para todo conjunto abierto $O \subset Y$.

Bajo algunas condiciones adicionales, las funciones casi abiertas llegan a ser abiertas.

Definición A.14. Decimos que una función $f: X \rightarrow Y$ es localmente cerrada en un punto $x \in X$, si para todo conjunto abierto $U \subset X$, con $x \in U$, existe una vecindad $N$ de $x$ en $X$ tal que $N \subset U$ y $f(N)$ es cerrada en $Y$. Si $f$ es localmente cerrada en todo punto de $X$, decimos que $f$ es localmente cerrada.

Proposición A.15. Toda función casi abierta localmente cerrada $f: X \rightarrow Y$ es abierta.

Corolario A.16. Toda función casi abierta de un espacio localmente pseudocompacto Tychonoff $X$ a un espacio regular $Y$, de pseudocaracter numerable, es abierta.

Corolario A.17. Si $X$ y $Y$ son espacios Hausdorff. Si $X$ es localmente compacto, entonces toda función casi abierta de $X$ a $Y$ es abierta.

Corolario A.18. Toda función cerrada casi abierta es abierta. En particular, las funciones casi abiertas entre espacios compactos Hausdorff son abiertas. 


\section{A.2. Acciones continuas de grupos topológi- cos sobre espacios}

En está sección se establece el concepto de acción de un grupo sobre un espacio topológico. Defininiremos este concepto en toda su generalidad y estableceremos algunos hechos generales que se aplican en la siguiente sección para el estudio de la estructura de los espacios Hausdorff compactos que admiten una acción transitiva de un grupo topológico $\omega$-estrecho.

Definición A.19. Sea $X$ un conjunto no vacío, y $G$ un grupo abstracto con identidad $e$. Supongamos que una función $\theta: G \times X \rightarrow X$ satisface las siguientes condiciones para todos $g, h \in G$ y $x \in X$ :

(A1) $\theta(e, x)=x$

(A2) $\theta(h, \theta(g, x))=\theta(h g, x)$

Entonces decimos que $G$ actúa sobre $X$ y que $\theta$ es una acción (izquierda) de $G$ sobre $X$. En la práctica, usualmente escribimos $g x($ o $g * x)$, en lugar de, $\theta(g, x)$. Si $\theta$ es una acción de un grupo $G$ sobre un conjunto $X$, todo elemento $g \in G$ determina una traslación $\theta_{g}$ de $X$, definida por, $\theta_{g}(x)=\theta(g, x)$, para cada $x \in X$ o, equivalentemente, $\theta_{g}(x)=g x$. Se sigue de $(A 1)$ y $(A 2)$ que $\theta_{g}$ es una biyección de $X$, para cada $g \in G$. De hecho, $\theta_{e}$ es la función identidad de $X$ en sí mismo, y $\theta_{h g}=\theta_{h} \circ \theta_{g}$ para cualesquiera $g, h \in G$. Por lo tanto, $\left(\theta_{g}\right)^{-1}=\theta_{g^{-1}}$, esto es, la función inversa de $\theta_{g}$ es $\theta_{g^{-1}}$, lo cual implica que $\theta_{g}$ es una biyección de $X$.

Definición A.20. Supongamos que $H \subset G$ y $A \subset X$ son conjuntos no vacíos, y que $x \in X$. Sea

$$
H A=\{h a: h \in H, a \in A\} \text { y } H x=\{h x: h \in H\} .
$$

Decimos que $A$ es $H$-invariante si $H A \subset A$. Por abreviar, los subconjuntos $G$-invariantes de $X$ son llamados invariantes. La órbita de un punto $x \in X$ es el conjunto $G x$. Se sigue de $(A 1)$ y $(A 2)$ que $G x$ es un subconjunto invariante de $X$ que contiene a $x$. Claramente, todo subconjunto invariante $A$ de $X$ es la unión de las órbitas de elementos de $A$.

Dados $x, y \in X$, escribimos $x \sim y$ si $y$ está en la órbita de $x$ bajo la acción del grupo $G$. Vamos a demostrar que la relación binaria $\sim$ sobre X determina la partición de $X$ en las órbitas. 
Proposición A.21. La relación $\sim$ es una relación de equivalencia sobre $X$. Las clases de equivalencia $[x]$ de un elemento arbitrario $x \in X$, con respecto $a \sim$, es la órbita $G x$.

En la proposición anterior, podemos definir el conjunto cociente

$$
X / G=\{[x]: x \in X\}
$$

Llamado el conjunto de órbitas. La función natural cociente $\pi: X \rightarrow X / G$ definida por $\pi(x)=[x]$, para cada $x \in X$, es llamada la proyección orbital. Es fácil verificar que un conjunto $A \subset X$ es invariante si y sólo si $A=\pi^{-1} \pi(A)$. Para todo $x \in X$, el conjunto

$$
G_{x}=\{g \in G: g x=x\}
$$

es llamado el estabilizador de $x$. Dos propiedades importantes del estabilizador están dadas en la siguiente proposición.

Proposición A.22. El estabilizador $G_{x}$ de cualquier punto $x \in X$ es un subgrupo de $G$. Además, $G_{g x}=g G_{x} g^{-1}$ para todo $g \in G$.

Corolario A.23. El estabilizador $G_{x}$ es un subgrupo invariante de $G$ si $y$ sólo si $G_{x}=G_{y}$, para todo $y \in G x$.

Definición A.24. Una acción $\theta: G \times X \rightarrow X$, es llamada:

- Libre si $G_{x}=\{\mathrm{e}\}$, para cada $x \in X$, o, equivalentemente, la igualdad $g x=x$ sólo es válido para $g=e$. En otras palabras, la traslación $\theta_{g}$ de $X$ no tiene puntos fijos si $g \neq e$.

- Eficaz, si $\bigcap_{x \in X} G_{x}=\{e\}$.

- Transitiva, si $G x=X$ para cada $x \in X$.

Definición A.25. Sea $G$ un grupo topológico y $X$ un espacio topológico. Una acción $\theta$ de $G$ sobre $X$ es llamada continua si $\theta$ es continua como una función de $G \times X$ en $X$. El espacio $X$ es llamado un $G$-espacio.

Proposición A.26. Toda acción continua $\theta: G \times X \rightarrow X$ de un grupo topológico $G$ sobre un espacio $X$ es una función abierta. 
Similarmente, en el caso de homomorfismos de grupos topológicos, la continuidad de una acción $\theta$ de un grupo topológico $G$ la podemos deducir de la continuidad en la identidad de $G$.

Proposición A.27. La continuidad de una acción $\theta: G \times X \rightarrow X$ de un grupo topológico $G$ con identidad e sobre un espacio $X$ es equivalente a la continuidad de $\theta$ en los puntos del conjunto $\{e\} \times X \subset G \times X$.

Proposición A.28. Si $\theta: G \times X \rightarrow X$ es una acción continua de un grupo topológico $G$ sobre un espacio $X$, entonces la proyección orbital $\pi: X \rightarrow$ $X / G$ es abierta.

Cuando $G$ es un grupo compacto, la conclusión de la proposición anterior se puede reforzar considerablemente.

Teorema A.29. Si un grupo topológico compacto $H$ actúa continuamente sobre un espacio Hausdorff, entonces la proyección órbital $\pi: X \rightarrow X / G$ es una función perfecta y abierta.

Definición A.30. Si $X$ y $Y$ son $G$-espacios con acciones continuas $\theta_{X}: G \times$ $X \rightarrow X$ y $\theta_{Y}: G \times Y \rightarrow Y$. Una función continua $f: X \rightarrow Y$ es llamada $G$-equivariante (o equivariante) si $\theta_{Y}(g, f(x))=f\left(\theta_{X}(g, x)\right)$, esto es, $g f(x)=$ $f(g x)$, para todo $g \in G$ y todo $x \in X$.

Si $\eta=\left\{X_{i}: i \in I\right\}$ es una familia de $G$-espacios, entonces el espacio producto $X=\prod_{i \in I} X_{i}$ tiene una estructura natural de un $G$-espacio. Definimos la acción de $G$ sobre $X$, tomando cualquier $g \in G$ y cualquier $x=\left(x_{i}\right)_{i \in I} \in X$, y haciendo $g x=\left(g x_{i}\right)_{i \in I}$. Así, $G$ actúa sobre $X$ coordenada a coordenada.

Proposición A.31. La acción coordenada a coordenada de G sobre el producto $X=\prod_{i \in I} X_{i}$ de $G$-espacios es continua, esto es, $X$ es un $G$-espacio.

\section{A.3. Teoremas de Uspenskij's sobre accio- nes transitivas continuas de grupos $\omega$ - estrechos sobre compactos}

En esta sección, hacemos uso de las técnicas desarolladas en secciones anteriores. 
Proposición A.32. Supongamos que un grupo topológico w-estrecho $G$ actúa continuamente y transitivamente sobre un espacio $Y$ con la propiedad de Baire. Entonces, para cualquier $y \in Y$, la función $\theta^{y}: G \rightarrow Y$ definida por $\theta^{y}(g)=$ gy, para cada $g \in G$, es casi abierta.

Proposición A.33. Supongamos que un grupo topológico $\omega$-estrecho $G$ actúa continuamente sobre un espacio métrico $(X, d)$ por isometrías. Entonces la órbita $G x$ es separable, para cada $x \in X$.

Definición A.34. Sea $\theta_{X}: G \times X \rightarrow X$ una acción continua de un grupo topológico $G$ sobre un espacio compacto $X$. Denotamos por $C(X)$ al espacio de todas las funciones continuas de valores reales sobre $X$ dotado con la topología generada por sup-norm : $\|f\|=\sup _{x \in X}|f(x)|$, para cada $f \in$ $C(X)$.

Lema A.35. Si $X$ es un espacio compacto y $C(X)$ es el espacio de todas las funciones continuas de valores reales sobre $X$ con la métrica sup-norm. Si $F$ es un espacio separable de $C(X)$ y $\varphi$ es el producto diagonal de las funciones en $F$, entonces la imagen $\varphi(X)$ es compacta y metrizable.

El siguiente teorema es uno de los resultados principales de esta sección.

Teorema A.36 (V. V. Uspenskij). Si un espacio compacto $X$ admite una acción transitiva continua de un grupo topológico $\omega$-estrecho $G$, entonces $X$ es Dugundji. En particular, $X$ es diádico.

Teorema A.37 (V. V. Uspenskij). Si H es un subgrupo cerrado de un grupo topológico $\omega$-balanceado. Entonces todo conjunto $G_{\delta}$ en el espacio cociente G/H es Dugundji.

El siguiente teorema nos proporciona una gran variedad de ejemplos naturales de grupos topológicos que no son $\omega$-balanceados.

Teorema A.38. Supongamos que $X$ es un espacio compacto homogéneo cero-dimensional tal que el grupo Homeo $(X)$ de todos los homeomorfismo de $X$ sobre sí mismo, con la topología compacto-abierta, es w-balanceado. Entonces $X$ es Dugundji.

Como un colorario tenemos lo siguiente.

Corolario A.39. Supongamos que $X$ es un espacio compacto homogéneo cero-dimensional con estrechez numerable tal que el grupo Homeo $(X)$ de todos los homeomorfismos de $X$ sobre sí mismo, con la topología compactoabierta, es $\omega$-balanceado. Entonces $X$ es metrizable. 


\section{Bibliografía}

[1] Arhangel'skii. A., Classes of topological groups, Russian Math. Surveys 36 No 3 (1981), 151-174. Original en ruso en : Uspekhi Mat. Nauk 36 (1981), 127-146.

[2] Arhangel'skii. A., Continuous mappings, factorization theorems and function spaces, Proc. Moscow Math. Soc. 47 (1984), 1-22. Original en ruso en: Trudy Mosk. Mat. Obsch. 47 (1984), 3-21.

[3] Arhangel'skii. A., Topological groups and C-embeddings, Topol. Appl. 115 No 3 (2001), 265-289.

[4] Arhangel'skii. A. y Tkachenko. M., Topological Groups and Related Structures, Atlantis Press, 2008.

[5] Arhangel'skii. A. y Uspenskij. V., Topological groups: local versus global, Appl. General Topology 7 No 1 (2006), 67-72.

[6] Bruguera. M y Tkachenko. M., Bounded sets in topological groups and embeddings, Topol. Appl. 154 No 7 (2007), 1298-1306.

[7] Engelking. R., General Topology, Heldermann Verlag, Berlin, 1989.

[8] Gillman. L. y Jerison. M., Rings of Continuous Functions,Springer Verlag, Berlin, 1976.

[9] Guran. I., On topological groups close to being Lindelöf, Soviet Math. Dokl, 23 (1981), 173-175.

[10] Hewitt. E. y Ross. K., Abstract Harmonic Analysis,Springer-Verlag, New York, 1963. 
[11] Kats. G., An isomorphic mapping of topological groups into a direct product of groups satisfying the first axiom of countability, Uspekhi Mat. Nauk 8 No 6 (1953), 107-113 (en ruso).

[12] Kelley. J., General Topology, Springer-Verlag, New York, 1975.

[13] Pontryagin. L., Topological Groups, Gordon and Breach, Science Publishers, inc, New York, 1966.

[14] Tkachenko. M, Villegas. L, Hernández. C y Rendón. O., Grupos Topológicos, Lisbros de texto, manuales de prácticas y antologías, México, 1997. 